\title{
An exact theory of nonlinear waves on a Lagrangian-mean flow
}

\author{
By D. G. ANDREWS \\ U.K. Universities' Atmospheric Modelling Group, \\ Reading, Berkshire, England $\dagger$ \\ AND M. E. MCINTYRE \\ Department of Applied Mathematics and Theoretical Physics, \\ University of Cambridge \\ (Received 9 October 1976 and in revised form $12 \mathrm{July} \mathrm{1978)}$
}

An exact and very general Lagrangian-mean description of the back effect of oscillatory disturbances upon the mean state is given. The basic formalism applies to any problem whose governing equations are given in the usual Eulerian form, and irrespective of whether spatial, temporal, ensemble, or 'two-timing' averages are appropriate. The generalized Lagrangian-mean velocity cannot be defined exactly as the 'mean following a single fluid particle', but in cases where spatial averages are taken can easily be visualized, for instance, as the motion of the centre of mass of a tube of fluid particles which lay along the direction of averaging in a hypothetical initial state of no disturbance.

The equations for the Lagrangian-mean flow are more useful than their Eulerianmean counterparts in significant respects, for instance in explicitly representing the effect upon mean-flow evolution of wave dissipation or forcing. Applications to irrotational acoustic or water waves, and to astrogeophysical problems of waves on axisymmetric mean flows are discussed. In the latter context the equations embody generalizations of the Eliassen-Palm and Charney-Drazin theorems showing the effects on the mean flow of departures from steady, conservative waves, for arbitrary, finite-amplitude disturbances to a stratified, rotating fluid, with allowance for selfgravitation as well as for an external gravitational field.

The equations show generally how the pseudomomentum (or wave 'momentum') enters problems of mean-flow evolution. They also indicate the extent to which the net effect of the waves on the mean flow can be described by a 'radiation stress', and provide a general framework for explaining the asymmetry of radiation-stress tensors along the lines proposed by Jones (1973).

† Present address: Meteorology Department, Massachusetts Institute of Technology, Cambridge. 


\section{CONTENTS}

1. Introduction

page 611

2. The generalized Lagrangian-mean flow

2.1. Eulerian averaging

2.2. The generalized Lagrangian-mean operator $\overline{()^{\mathrm{L}}}$

2.3. Basic properties of $\overline{()^{\mathrm{L}}}$

2.4. Stokes corrections

3. Basic theorems governing mean-flow evolution

4. Mass conservation

4.1. The Jacobian $J$ and the mean-flow density $\tilde{\rho}$

4.2. Condition at an impermeable boundary

4.3. $\overrightarrow{\mathrm{u}}^{\mathrm{I}}$ as the motion of the centre of mass of a tube of fluid 625

5. The mean-flow equations and some of their general implications 626

5.1. A complete set of equations $\quad 626$

5.2. Longitudinally symmetric mean flow $\quad \mathbf{6 2 6}$

$\begin{array}{ll}\text { 5.3. Transient, conservative waves } & 628\end{array}$

5.4. Resonant forcing of the mean flow $\quad 629$

$\begin{array}{ll}\text { 6. On irrotational waves } & 630\end{array}$

7. The frozen-field transformation, and $\overrightarrow{\mathrm{u}}^{\mathrm{L}}-\mathrm{p}$ for irrotational flow 632

8. On the radiation-stress concept 633

8.1. A form of the mean-flow equations analogous to the Eulerian-mean equations 633

8.2. The physical meaning of $R_{i j}$

8.3. The asymmetry of $R_{i j} \quad 634$

8.4. $R_{i j}$ and the radiation-stress concept $\quad 634$

8.5. Relation to earlier theories $\quad 636$

9. Approximate equations for incompressible, Boussinesq flow, and the model of equatorial planetary waves on a beta-plane studied in $I$

$\begin{array}{ll}\text { 10. Concluding remarks } & 639\end{array}$

Appendix A. Manipulations involving the Jacobian $J$ and its cofactors $K_{i s} \quad 640$

$\begin{array}{lr}\text { A 1. Direct proof that } \tilde{\rho} \text { satisfies (4.2) } & 640\end{array}$

A 2. Direct proof that $\tilde{\rho}$ is a mean quantity $\quad 640$

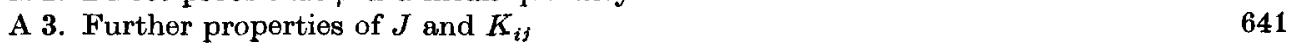

Appendix B. Derivation of theorem I $\quad 642$

Appendix C. Proof that $\xi$ returns to zero when the Eulerian disturbance returns

to zero, under the circumstances envisaged in $\S 5.3$ 


\section{Introduction}

Ever since Rayleigh (1896) drew attention to the role of viscosity in acoustic streaming, and Brillouin $(1925,1936)$ gave his penetrating analysis of the nature of acoustic radiation stresses, there have come to light an increasing number of interesting phenomena attributable to nonlinear rectification of oscillatory disturbances in fluids. Particularly impressive is the recent evidence that such effects drastically modify the large-scale distribution of azimuthal velocity in the earth's stratosphere (e.g. Holton 1975; Lindzen \& Tsay 1975). The waves concerned involve buoyancy and Coriolis effects. Nonlinear rectification of such waves may also be responsible for the four-day rotation of Venus' stratosphere (Fels \& Lindzen 1974; Plumb 1975) and could play a role in determining differential rotation in stellar interiors (E. A. Spiegel, personal communication). In these astrogeophysical examples the wave-induced stresses cause the mean flow at one height and latitude to undergo systematic longitudinal acceleration at the expense of a corresponding deceleration (or a wave drag on topography) at another height and latitude. The resulting feedback on the waves themselves can, in some cases, give rise to interesting time-dependent effects; for instance the whole wave, mean-flow system may 'vacillate' on a time scale related to wave amplitude (e.g. Holton \& Mass 1976; Plumb 1977; Holton \& Dunkerton 1978); perhaps the best documented examples are the 'quasi-biennial oscillation' of the zonal wind in the earth's equatorial stratosphere (Plumb 1977 and references therein) and a recent laboratory simulation thereof (Plumb \& McEwan 1978).

We have shown elsewhere how generalizations of the results of Eliassen \& Palm (1961), Charney \& Drazin (1961) and others furnish a powerful aid to the theoretical description of such wave, mean-flow interaction phenomena, in particular exhibiting in a very general way how mean-flow acceleration is linked to wave transience, dissipation, and excitation (Andrews \& MeIntyre 1976a, b, 1978a, hereafter called I-III; see also the related work by Boyd 1976 and the review articles by McIntyre 1977, 1978). Earlier, Braginskii (1964) and Soward (1972) had obtained comparable results in the context of dynamo theory. The most natural way of expressing these and other results on rectified wave effects - for example the case of acoustic streaming shown in figure 3 below - appears to be in terms of the Lagrangian-mean flow. However, the concept of 'Lagrangian mean' is often required in a more general sense than its classical sense of the mean following a single fluid particle; and the question of formulation which thus arises is the first concern of this paper.

An important step towards the required general concept was provided by the transformation theory of the Lagrangian equations of motion given, for instance, by Frieman \& Rotenberg (1960) and more systematically by Eckart (1963). Its application to problems of mean-flow evolution has been developed with great ingenuity by Dewar (1970), Bretherton (1971) and Soward (op. cit.; see also Soward \& Roberts 1976; Moffatt 1978). However, all these developments either depended at some stage on various asymptotic approximations or (in the case of Soward's work) were exact in principle but did not lead to exact theorems on mean-flow evolution due to finiteamplitude waves. The question thus remained as to whether there is an underlying exact theory, which in some natural sense generalizes the classical, approximate notion of Lagrangian-mean flow, and which unifies and extends all the foregoing results. 
The purpose of this paper is to construct such a theory, and to set it in the perspective of earlier work. In $\S \S 2$ and 3 we show that out of the infinite family of transformations considered by Eckart there is a natural choice which leads to a simple yet exact definition of the generalized Lagrangian-mean velocity $\overline{\mathbf{u}}^{\mathrm{L}}$, and to finite-amplitude versions of the basic theorems on mean-flow evolution. The transformation in question is not a member of the class considered by Soward (1972). The resulting 'generalized Lagrangian-mean (GLM) description' of wave, mean-flow interaction does, however, contain the theories of Bretherton, Dewar (op. cit.) and Grimshaw (1975) (which are based on 'two-timing' expansions for slowly-varying, almost-sinusoidal waves); so our theory may be thought of as an exact counterpart to those approximate theories.

Along with its predecessors, the -GLM description is really a hybrid (EulerianLagrangian) description of wave, mean-flow interaction, since it describes the generalized Lagrangian-mean flow by means of equations in Eulerian form, with position $\mathbf{x}$ and time $t$ as independent variables. An Eulerian description of the Lagrangian-mean flow is desirable because ideas like 'steady mean flow' often need to be expressed. Equally important are ideas like steadiness of the wave field, or its spatial homogeneity in some direction; so the particle displacement $\xi$ associated with the waves is likewise defined as a function of $\mathbf{x}$ and $t$ and not primarily as a function of the individual particle label as in a purely Lagrangian description. (Of course the definition of $\boldsymbol{\xi}(\mathbf{x}, t)$ at finite amplitude is itself a non-trivial part of our problem.) An interesting by-product of the approach is that it leads to what may be the first exact definition of pseudomomentum, or wave 'momentum'. The hybrid, Eulerian-Lagrangian character of the formulation seems essential to this, owing to the nature of the translational symmetry operation associated with conservation of pseudomomentum (Peierls 1976). More details are given in the following paper (Andrews \& McIntyre $1978 b$ ), where the related entities of wave-action and pseudoenergy are also discussed. $\dagger$

The pseudomomentum p per unit mass enters problems of mean-flow evolution in a way generally expressed by the basic theorems already mentioned, which are given in §3. An interesting special case is that of irrotational flow, as in the usual theories of acoustic or surface-gravity waves, where $p$ enters in a particularly simple way. This becomes apparent in $\S \S 3$ and 7 , where, following lines of argument suggested by the work of Bretherton (1971) and Soward (1972), we find that $\overline{\mathbf{u}}^{\mathrm{L}}-\mathbf{p}$ is exactly irrotational whenever the total motion is irrotational. In $\$ 6$ we show how this applies to a simple example of inviscid acoustic streaming (figure 3). The irrotationality of $\overline{\mathbf{u}}^{\mathrm{L}}-\mathbf{p}$ explains why it sometimes turns out that $\overline{\mathbf{u}}^{\mathrm{L}}=\mathbf{p}$ in irrotational-wave problems. Our example, however, has distributions of $\overline{\mathbf{u}}^{\mathrm{I}}$ and $\mathbf{p}$ which are entirely different from each other, even far from the wave source; this is due to the different boundary conditions which they satisfy at the wave source, and is typical of most problems with non-trivial boundary conditions.

In $\S \S 4$ and 5 a complete set of equations for the mean flow is derived for the general

$\dagger$ For linear, slowly-varying waves - more precisely, under those circumstances for which Bretherton \& Garrett's (1968) definition of intrinsic wave-energy density $E$ holds - pseudomomentum density is approximately equal to divided by the intrinsic phase velocity [Peierls 1976; McIntyre 1977; Andrews \& McIntyre 1978b, equation (5.10)]. Our terminology follows established usage in solid-state physics and expresses the fact that pseudomomentum and momentum are different physical entities, corresponding to different translational symmetry operations. 
case of a stratified, rotating fluid under external and self-gravity, using the theorems of $\S 3$. The equations are used to discuss the role of wave dissipation, etc., in meanflow evolution, particularly for the important case of 'longitudinally symmetric' mean flow appropriate to the astrogeophysical examples mentioned earlier and studied in I-III. The equations also enable us to examine in $\$ 8$ the general limitations of the 'radiation-stress' concept, and the reasons why radiation-stress tensors are generally asymmetric (cf. Garrett 1968; Jones 1973). The importance of considering a complete set of mean-flow equations and boundary conditions hardly needs emphasis; for instance the rate at which mean momentum or angular momentum is removed from one height and latitude, and reappears at another, need not depend solely on the wave-induced flux of momentum appearing in the mean longitudinal momentum equation, a well-known fact illustrated in various ways by the examples of $\S 4.2$, $\S 8.4$ and $\S 9$ below. The importance of describing boundary effects carefully is illustrated in $\$ 4.2$, and also by the acoustic example already mentioned $(\$ 6)$. It is here that the GLM description has one of its characteristic advantages, in that the normal component of $\overline{\mathbf{u}}^{\mathrm{L}}$ must vanish even at a 'fluctuating' boundary, such as a wave maker, whose mean position is fixed $(\$ 4.2)$; a corresponding statement is seldom true of the Eulerian-mean flow $\overline{\mathbf{u}}$.

In $\S 9$ we note some points requiring special care when applying the GLM formalism to incompressible or Boussinesq flow, and to motion 'on a beta-plane'. These points are illustrated by reference to the model of equatorial planetary waves studied in $\mathrm{I}$.

At a late stage in developing the present ideas we learned of concurrent work by Bretherton (1979), in which he arrived at a generalized Lagrangian-mean description similar to ours. His and our work were largely independent, but comparing notes has led, we believe, to improvements in both formulations. The aims and results are complementary. Bretherton proceeds from a variational formulation and thereby exposes (via the example of infinitesimal waves on an axisymmetric mean flow) a close relationship between our results and the conservation laws for the 'energymomentum' tensor of classical field theory (Landau \& Lifshitz 1975). This relationship is further explored in the companion paper. In the present paper we omit variational considerations, and lay more emphasis on a systematic development of the elementary procedures for finite-amplitude disturbances, and on a general representation of the all-important departures from conservative motion. The definitions and results on finite-amplitude disturbances (with the exception of Kelvin's circulation theorem for conservative flow; see $\S 3$ and Bretherton 1979) have not to our knowledge been given elsewhere.

\section{The generalized Lagrangian-mean flow}

\subsection{Eulerian averaging}

A single basic formalism underlies all the different applications in which spatial, temporal, ensemble, or 'two-time' averaging are used. To bring this out economically we shall develop the theory in a somewhat abstract way, from time to time referring to special cases to help fix ideas. It is necessary to begin with a formal statement of what is meant in general by the usual Eulerian averaging operator; the generalized Lagrangian-mean operator will then be introduced in $\$ 2.2$. 
Suppose that the dependent variables of the problem are expressed as real-valued scalar or tensor fields $\varphi(\mathbf{x}, t), \psi(\mathbf{x}, t), \ldots$, where $\mathbf{x}$ is position and $t$ time; these will include the usual pressure, density and velocity fields. Let $(\overline{)}$ be a general averaging operator taking $\varphi(\mathbf{x}, t), \psi(\mathbf{x}, t), \ldots$ into corresponding (Eulerian-mean) fields $\bar{\varphi}(\mathbf{x}, t)$, $\bar{\psi}(\mathbf{x}, t), \ldots$. In the case of an ensemble average the fields will depend on a suitable label $\alpha$ as well as on $(\mathbf{x}, t)$, and ( will involve integration over $\alpha$. It is convenient to require that $\bar{\varphi}$ has the same tensor character as $\varphi$, and a notation such as $\vec{\varphi}_{i j}$ will mean a Cartesian component of the tensor $\bar{\varphi}$. The component $\bar{\varphi}_{i j}$ will be equal to the result of averaging a single component $\varphi_{i j}$ except in the case of an azimuthal or other nonrectilinear space average. $\dagger$

We assume that ( ) and the fields satisfy the following postulates, where $a$ and $b$ are constant scalar or isotropic tensor quantities, and products may or may not involve contraction:

(i) $\overline{a p+b \bar{\psi}}=a \bar{\varphi}+b \bar{\psi}$ (the operator $(\overline{\text { ) }}$ is linear),

(ii) $\bar{a}=a$,

(iii) $\overline{\bar{\varphi} \psi}=\bar{\varphi} \bar{\psi}$,

(iv) $T$ ) commutes with covariant differentation, i.e. with partial differentiation with respect to Cartesian co-ordinates $\left(x_{i}, t\right)$ : thus $\left[\overline{()_{, \mu}}=\left[\overline{()_{, \mu}}\right]\right.$ where ()$_{, \mu}$ denotes either $\partial() / \partial x_{i}$ or $\partial() / \partial t$.

(v) $\overline{\mathbf{x}}=\mathbf{x} ; \bar{t}=t$; and (i) and (iii) still hold when $\mathbf{x}$ or $t$ is substituted for $\varphi$ or $\psi$.

(vi) $\overline{|\varphi|^{2}} \geqslant 0$; and if $\overline{|\varphi|^{2}}=0$ at $\left(\mathbf{x}_{0}, t_{0}\right)$, then $\varphi\left(\mathbf{x}_{0}, t_{0}\right)=0$. (If $\varphi$ is a tensor field of rank greater than zero, then $|\varphi|^{2}$ means the sum $\varphi_{i j k . .} \varphi_{i j k \ldots}$ over all Cartesian components.)

Postulate ( $\mathbf{v}$ ) is a matter of definition and admits $\mathbf{x}$ and $t$ (which are co-ordinates, not tensor fields) into the class of entities upon which $(\overrightarrow{)}$ may operate. The postulate ensures for instance that the 'average position' of a motionless fluid particle is meaningful, and the same as its actual position.

Note that (ii) and (iii) imply that

$$
\overline{\bar{\varphi}}=\bar{\varphi}
$$

† A tensor definition of the azimuthal averaging operator which avoids reliance on cylindrical or other special co-ordinates is as follows. Let $\hat{\mathbf{z}}$ be a unit vector along the symmetry axis for the mean fields, and introduce the rotation tensor

$$
r_{i k}^{(\lambda)}=\delta_{i k} \cos \lambda+\hat{z}_{i} \hat{z}_{k k}(1-\cos \lambda)-\epsilon_{i k p} \hat{z}_{p} \sin \lambda
$$

For instance $r_{i k}^{(\lambda)} v_{k}$ is the $i$ th component of any vector $\mathbf{v}$ after it has been rotated through an angle $\lambda$ about the unit vector $\hat{\mathbf{z}}$. Then the azimuthal average $\bar{\varphi}_{i j k \ldots}(\mathbf{x}, t)$ of a general tensor field $\varphi_{i j k \ldots}(\mathbf{x}, t)$ is

$$
\frac{1}{2 \pi} \int_{0}^{2 \pi} r_{i p}^{(\lambda)} r_{j q}^{(\lambda)} r_{k s}^{(\lambda) \ldots} \varphi_{p a s . .}\left\{r_{l m}^{(-\lambda)}\left(x_{m}-x_{(0) m}\right), t\right\} d \lambda
$$

where $\mathbf{x}_{(0)}$ is any point on the symmetry axis. For each $\lambda$ the integrand is the tensor field obtained by evaluating the original field at the position rotated through $-\lambda$, and then rotating the resulting tensor back through the angle $\lambda$. If $\varphi$ is a scalar, i.e. a tensor of rank zero, then it is understood that no $r$ 's appear in front of $\varphi$ in the integrand. It is straightforward to verify that $\bar{\varphi}$ is axisymmetric (i.e. that the integrand with $\bar{\varphi}$ in place of $\varphi$ is independent of $\lambda$ ), and that ( ) satisfies all the postulates about to be stated. It can also be verified that the cylindrical components of $\bar{\varphi}$ are equal to the result of naively averaging the cylindrical components of $\varphi$, which of course is the familiar rule used in practice. 
Furthermore (i)-(iii) imply that

$$
\overline{a \bar{\varphi}+b \bar{\psi}}=a \bar{\varphi}+b \bar{\psi}, \quad \overline{\bar{\varphi}} \bar{\psi}=\bar{\varphi} \bar{\psi}, \quad \overline{1 / \bar{\varphi}}=1 / \bar{\varphi},
$$

and similarly that other functions of averaged quantities alone are themselves 'averages', i.e. are unaltered by being further averaged. We assume of course that the fields are suitably behaved functions, in a given context of averaging and other operations. Note for instance that a gravitational potential field $\Phi$ will usually have a well-defined spatial average with respect to a horizontal, but not the vertical, direction.

\subsection{The generalized Lagrangian-mean operator $\overline{()^{\mathrm{L}}}$}

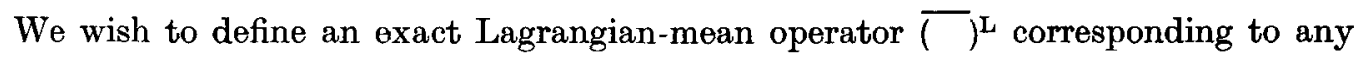
given Eulerian-mean operator $(\overrightarrow{)}$. To do this we must define with equal generality an exact, disturbance-associated particle displacement field $\xi(x, t)$. It will then be possible to write

$$
\overline{\varphi(\mathbf{x}, t)} \bar{L}=\overline{\varphi\{\mathbf{x}+\xi(\mathbf{x}, t), t}\}
$$

i.e. to define ()$^{\mathrm{L}}$ as the average taken with respect to the displaced positions $\mathbf{x}+\xi$, considered as functions of $\mathbf{x}$ and $t$.

As a preliminary, it is useful to recall two properties of the mapping

$$
\mathbf{x} \rightarrow \mathbf{x}+\xi(\mathbf{x}, t)
$$

for a general function $\xi(x, t)$, i.e. regarding it simply as a general transformation in the spirit of Eckart (1963) and Soward (1972). Introducing the notation

$$
\varphi^{\xi}(\mathbf{x}, t)=\varphi\{\mathbf{x}+\xi(\mathbf{x}, t), t\},
$$

we have first the chain-rule identities

where

$$
\left(\varphi^{\xi}\right)_{, t}=\left(\varphi_{, t}\right)^{\xi}+\left(\varphi_{, j}\right)^{\xi} \Xi_{j, t}, \quad\left(\varphi^{\xi}\right)_{, i}=\left(\varphi_{, j}\right)^{\xi} \Xi_{j, i}
$$

$$
\boldsymbol{\Xi}(\mathbf{x}, t)=\mathbf{x}+\boldsymbol{\xi}(\mathbf{x}, t)
$$

$(\quad)_{, t}=\partial(\quad) / \partial t$ and ()$_{, i}=\partial(\quad) / \partial x_{i}$. To obtain the second property we must make the following assumption:

(vii) The mapping (2.2) is invertible.

Then for any given $\mathbf{u}(\mathbf{x}, t)$ there is evidently a unique 'related velocity field' $\mathbf{v}(\mathbf{x}, t)$ such that when the point $\mathbf{x}$ moves with velocity $\mathbf{v}$ the point $\mathbf{x}+\xi$ moves with the actual fluid velocity $\mathbf{u}^{\xi}$, i.e.

An immediate consequence is

$$
(\partial / \partial t+\mathbf{v}, \nabla) \boldsymbol{\Xi}=\mathbf{u}^{\boldsymbol{\xi}}
$$

$$
(\partial / \partial t+\mathbf{v} \cdot \nabla)\left(\varphi^{\xi}\right)=(D \varphi / D t)^{\xi}
$$

for any field $\varphi$, where $D / D t=\partial / \partial t+\mathbf{u} . \nabla$ [Eckart 1963, equation (3.5); Bretherton 1971, p. 87; Soward 1972, equation (1.34)].

Now the GLM description is obtained by requiring that

and

$$
\begin{gathered}
\overline{\xi(\mathrm{x}, t)}=0, \\
\overline{\mathrm{v}(\mathbf{x}, t)}=\mathrm{v}(\mathbf{x}, t),
\end{gathered}
$$

i.e. that $\xi$ is a true disturbance quantity and $\mathbf{v}$ a mean quantity. It will then follow, by applying the operator $(\overline{)}$ to $(2.6 a)$ and using postulates (i) and (iii) $-(\mathbf{v})$ and 


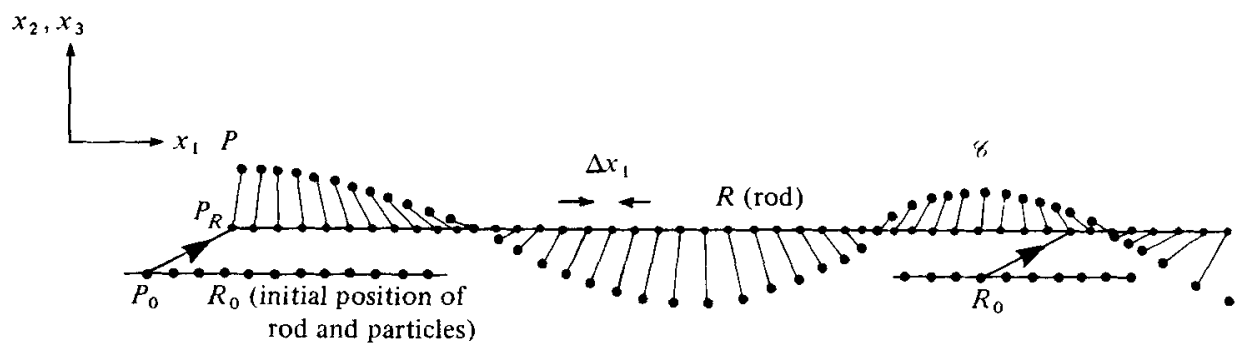

(a)

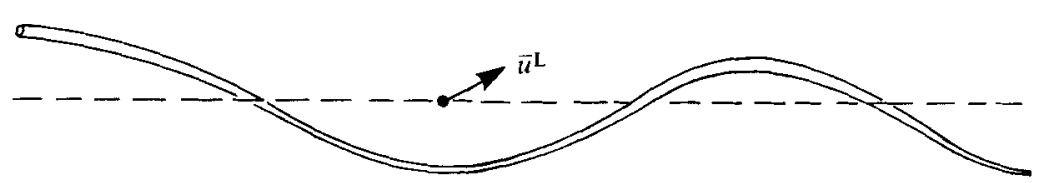

(b)

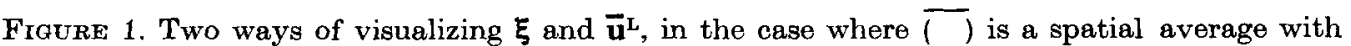
respect to $x_{1}$. (a) Mechanical analogy in which a rigid rod $R$ moves with velocity $\overline{\mathbf{u}}^{\mathrm{L}}$ under the pull of a large number of elastic bands whose lengths and orientations give $\xi$ at each point on $R$ (see text below). (b) A material tube $\mathscr{V}^{\widetilde{\xi}}$ of fluid whose centre of mass moves with velocity $\overline{\mathbf{u}}^{\mathrm{L}}$, in the limit of infinite tube length, as conjectured by Matsuno (see text, $§ 4.3$ ).

definitions (2.1) and (2.3) above, that $\mathrm{v}=\overline{\mathbf{u}}^{\mathrm{L}}$, so that in terms of a 'Lagrangian-mean material derivative'

$$
\bar{D}^{\mathrm{L}}=\partial / \partial t+\overline{\mathbf{u}}^{\mathrm{L}} \cdot \nabla
$$

the relation $(2.6 a)$ may be rewritten as

or equivalently

where

$$
\begin{aligned}
& \bar{D}^{\mathrm{L}} \mathbf{\Xi}=\mathbf{u}^{\xi}, \\
& \bar{D}^{\mathrm{L} \xi}=\mathbf{u}^{\mathbf{l}}, \\
& \mathbf{u}^{l}(\mathbf{x}, t)=\mathbf{u}^{\boldsymbol{\xi}}-\overline{\mathbf{u}}^{\mathbf{L}} .
\end{aligned}
$$

The quantity $\mathbf{u}^{l}$ will be called the Lagrangian disturbance velocity; we evidently have

$$
\overline{\mathbf{u}^{l}}=0 \text {. }
$$

It is (2.7) and (2.12), which characterize $\xi$ and $\mathbf{u}^{l}$ as disturbance-associated fields, and $(2.10 b)$, which states that $\mathbf{u}^{l}$ is equal to the mean material rate of change of $\xi$, which will make it legitimate to regard $\xi$ as a disturbance-associated particle displacement. It will turn out that the requirements (2.7) and (2.8), and their consequences (2.10) and (2.12), put (2.2) outside the class of transformations considered by Soward (1972). Those requirements, however, underlie the remarkable analytical simplicity of many of the subsequent developments in this and the companion paper. The aim is to enable 'wave' and 'mean' effects to be separated from one another as cleanly as possible at finite amplitude. $\dagger$

The self-consistency of the requirements (2.7) and (2.8) may be made immediately plausible by means of a mechanical analogy which holds for the case where $(\overline{)}$ is a

+ Soward (personal communication) points out that it would have been possible to impose one of (2.7) or (2.8) within his formulation. The reason why both cannot be adopted is a restriction he imposes on the Jacobian $J=\operatorname{det} \Xi_{i, j}$; see below for details [(9.3) et seq.]. 
spatial average involving integration along a line $R$ parallel to the $x_{1}$ axis, as would be appropriate for studying waves or instabilities on parallel shear flows, in which case $\overline{()^{\mathrm{L}}}$ is an average along the wavy line $\mathscr{C}$ defined at any instant by applying to $R$ the mapping (2.2). + Suppose that there is no disturbance anywhere at some initial time $t=t_{0}$. In figure $1(a)$, let $R_{0}$ be a line parallel to the $x_{1}$ axis. Fix attention on a row of marked particles which are initially spaced at equal distances $\Delta x_{1}$ along $R_{0}$, and then watch these particles as they follow the fluid motion. In the mechanical analogy (which has no dynamical connexion with the fluid motion) we imagine that a thin, light, rigid rod $R$ initially coincides with $R_{0}$, but is subsequently free to move while remaining parallel to the $x_{1}$ axis. The position $P$ of a typical particle of fluid whose initial position was $P_{0}$ is joined to the point $P_{R}$ on $R$ which initially coincided with $P_{0}$; the ligaments joining the marked particles to $R$ consist of identical 'elastic bands' such that $P_{R}$ is pulled towards $P$ with a force proportional to the distance $P_{R} P$, and similarly for the other points. The rod $R$ is imagined to be in static equilibrium under the pull of all the ligaments. Then, in the limit $\Delta x_{1} \rightarrow 0$, the rod will move with velocity $\overline{\mathbf{u}}^{\mathrm{L}}$; and if $\mathbf{x}$ is the current position of $P_{R}, \xi(\mathbf{x}, t)$ is the 'elastic-band vector' $\overrightarrow{P_{R} P}$. The static equilibrium of the rod expresses the requirement (2.7), and the rigidity of the rod expresses (2.8).

It will turn out $(\S 4.3)$ that $\overline{\mathbf{u}}^{\mathrm{L}}$ is also exactly equal to the velocity of the centre of mass of a thin tube of fluid initially lying in the $x_{1}$ direction (figure $1 b$ ). This result was conjectured by T. Matsuno (personal communication) on the basis of a calculation for small disturbance amplitude.

In order to give a formal definition of $\xi$ and $\overline{\mathbf{u}}^{\mathrm{L}}$ for general $(\overrightarrow{)}$ it will be convenient to introduce a further postulate:

(viii) Each Lagrangian-mean trajectory (solution of $d \mathbf{x} / d t=\overline{\mathbf{u}}^{\mathrm{L}}(\mathbf{x}, t)$ ) passes through at least one point $\left(\mathbf{x}_{0}, t_{0}\right)$ in the neighbourhood of which there is no disturbance, in the sense that the mean square of $\xi$ and of every other disturbance field $\varphi^{\prime}$ is zero:

$$
\overline{|\xi|^{2}}=\overline{\left|\varphi^{\prime}\right|^{2}}=0 \text {. }
$$

For instance the $t_{0}$ for each trajectory might be a single, initial time at which there is no disturbance anywhere, as just envisaged. The growth of the disturbance from zero need only be hypothetical, of course; all that is strictly necessary is that the setting up of the disturbance in this way should be kinematically possible (with mass conserved - a requirement whose importance will become apparent in $\S 4$ ).

Note the immediate implication [from (vi) and (viii)] that

$$
\left.\boldsymbol{\xi}\right|_{\mathbf{x}=\mathbf{x}_{0}, t=t_{\mathrm{n}}}=0 .
$$

In the case of an ensemble average (2.13) means that $\xi\left(x_{0}, t_{0} ; \alpha\right)=0$ for all $\alpha$, i.e. for each member of the ensemble. An equivalent postulate is implicit in the Lagrangian labelling procedure used by Bretherton (1979).

We may now define $\xi$ and $\overline{\mathbf{u}}^{\mathrm{L}}$ via integration of $(2.10 b)$ along mean trajectories, a sufficient set of which is taken at a time to allow ( ) to be computed at each time step. The initial condition on $\xi$ is supplied by (2.13). Thus $(2.10 b)$ is now satisfied by

$\dagger$ Note that the average is not uniformly weighted with respect to arc length $s$ along $\mathscr{C}$, since (2.1) involves integration with respect to $d x$ and not $d s$. 
definition; the other key property (2.7) follows in a similar way from (viii) and the earlier postulates, including (vi) $\dagger$

\subsection{Basic properties of $\overline{()^{\mathrm{L}}}$}

One reason why the Lagrangian-mean operator is useful is that it gives simple results when applied to the material derivative. The general kinematical relation $(2.6 b)$ now becomes

$$
(D \varphi / D t)^{\xi}=\bar{D}^{\mathrm{L}}\left(\varphi^{\boldsymbol{\xi}}\right) .
$$

Because the velocity field involved in $\bar{D}^{\mathrm{L}}$ is a mean field, (2.14) has two important corollaries:

and

$$
\overline{(\overline{D \varphi} / \overline{D t})^{\mathrm{L}}}=\bar{D}^{\mathrm{L}} \bar{\varphi}^{\mathrm{L}}
$$

where

$$
\left(D_{\varphi} / D t\right)^{l}=\bar{D}^{\mathrm{L}} \varphi^{l}
$$

$$
\left.\varphi^{l}=\varphi^{\xi}-\bar{\varphi}^{\mathrm{L}} \quad \overline{\left(\varphi^{l}\right.}=0\right),
$$

and where we have used (2.1), (2.3), and postulates (i), (iii) and (iv). It is noteworthy that (2.15) and (2.16) have much simpler forms than their more familiar, Eulerian counterparts

and

$$
\overline{D \varphi / D} t=\bar{D} \bar{\varphi}+\overline{\mathbf{u}^{\prime} \cdot \bar{\nabla} \varphi^{\prime}}
$$

$$
(D \varphi / D t)^{\prime}=\bar{D} \varphi^{\prime}+\mathbf{u}^{\prime} \cdot \nabla \bar{\varphi}+\mathbf{u}^{\prime} \cdot \nabla \varphi^{\prime}-\overline{\mathbf{u}^{\prime} \cdot \nabla \varphi^{\prime}},
$$

where $\bar{D}=\partial / \partial t+\overline{\mathbf{u}} \cdot \nabla$ and $\varphi^{\prime}$ denotes the Eulerian disturbance field $\varphi-\bar{\varphi}$.

Because of (2.15), application of the operator $\overline{()^{\mathrm{L}}}$ to any equation governing the total motion and involving a material rate of change, say

$$
D S / D t+Q=0,
$$

immediately generates a similar equation for the evolution of the corresponding Lagrangian-mean quantity,

$$
\bar{D}^{L} \overline{\mathcal{S}}^{\mathrm{L}}+\bar{Q}^{\mathrm{L}}=0,
$$

with no extra term such as that on the right of $(2.18)$. If $S$ is either entropy or potential vorticity (Eliassen \& Kleinschmidt 1957; equation (5.15) below), then for inviscid, adiabatic flow $Q=0$ and we have

In that event we also have

$$
\bar{D}^{\mathrm{L}} \overline{\mathcal{S}}^{\mathrm{L}}=\mathbf{0}
$$

$$
\bar{S}^{\mathbf{L}}(\mathbf{x}, t)=S^{⿷},
$$

by integration of $\bar{D}^{L}\left(\bar{S}^{L}-S^{\xi}\right)=0$ along mean trajectories, using postulate (viii). That is, the generalized Lagrangian mean of any convected quantity $S$ is exactly equal to the value at $\mathrm{x}+\xi$ of $S$ itself. The strong constraint on the evolution of the mean flow

$\dagger$ First, note that

$$
\left.\bar{\xi}\right|_{x=x_{0}, t=t_{0}}=0
$$

(For, by (i)-(iii) and (vi), $0 \leqslant \overline{|\xi-\bar{\xi}|^{2}}=\overline{|\xi|^{2}}-\overline{2 \xi \cdot \bar{\xi}}+|\bar{\xi}|^{2}=\left.\bar{\xi}\right|^{2}-|\bar{\xi}|^{2}$, whence $|\bar{\xi}|^{2} \leqslant|\bar{\xi}|^{2}=0$ at $\left(\mathbf{x}_{0}, t_{0}\right)$, by (viii); hence $|\bar{\xi}|^{2}=0$ and so $\bar{\xi}=0$.) We now apply $\overline{()}$ to $(2.10 b)$ to show with the help of (i)-(iv) that $\bar{D}^{L \bar{\xi}}=0$ for all $\mathbf{x}$ and $t$; then integration along mean trajectories with the use of the foregoing relation as initial condition shows that $\bar{\xi}=0$ for all $\mathbf{x}$ and $t$, verifying (2.7). 
represented by (2.22) and (2.23) would not be evident from an Eulerian-mean description, in which the equation corresponding to (2.21) is (2.29) below. Examples of problems in which the difference between (2.29) and (2.21) is crucial, with $S=$ entropy, include guided internal gravity waves, and virtually all astrogeophysical problems involving Coriolis effects in stratified fluids (e.g. Matsuno 1971; Grimshaw 1975; McIntyre 1973, 1977, 1978). An example where it is illuminating to take $S$ to be the potential vorticity is that of shallow-water motion in a rotating 'ocean basin' (Moore 1970 and references therein); see also Grimshaw [op. cit., equation (4.29)]. Relations of the type (2.23) also imply restrictions on the time development of $\xi$ itself; an example of circumstances where this is useful will be encountered in $\S 5.3$.

The price paid for the great simplicity of $(2.15)$ and $(2.16)$ is that the operator ()$^{\mathrm{L}}$ does not commute with $\partial / \partial t$ or $\partial / \partial x_{i}$, as is evident from (2.1) and (2.4). However, as immediate consequences of (i)-(iv) we have

$$
\begin{gathered}
\overline{(a \varphi+b \psi)^{\mathrm{L}}}=a \bar{\varphi}^{\mathrm{L}}+b \bar{\psi}^{\mathrm{L}}, \\
\overline{\boldsymbol{a}}^{\mathrm{L}}=a, \quad \overline{\bar{\varphi}^{\mathrm{L}}}=\bar{\varphi}^{\mathrm{L}}, \quad \overline{\bar{D}^{\mathrm{L}} \varphi}=\bar{D}^{\mathrm{L}} \bar{\varphi} .
\end{gathered}
$$

\subsection{Stokes corrections}

The relationship with the conventional Eulerian-mean description is represented by the 'Stokes correction' to each mean field, now exactly definable as

$$
\bar{\varphi}^{\mathrm{S}}(\mathbf{x}, t)=\bar{\varphi}^{\mathrm{L}}(\mathbf{x}, t)-\bar{\varphi}(\mathbf{x}, t) \text {. }
$$

For small disturbance amplitude $a$, Taylor expansion of (2.3) shows that

$$
\varphi^{\xi}=\bar{\varphi}+\varphi^{\prime}+\xi_{j} \varphi_{, j}+\frac{1}{2} \xi_{j} \xi_{k} \bar{\varphi}_{, j k}+O\left(a^{3}\right),
$$

since $\bar{\varphi}+\varphi^{\prime}=\varphi(\mathbf{x}, t)$ and $\bar{\varphi}_{j k}=\varphi_{j k}+O(a)$. Application of the operator $\overline{(T}$ to (2.26) gives a result which may be written, noting that $\bar{\varphi}^{\mathrm{s}}=\overline{\varphi^{\xi}}-\bar{\varphi}$, as

$$
\bar{\varphi}^{\mathrm{S}}=\overline{\xi_{j} \varphi_{, j}^{\prime}}+\frac{1}{2} \bar{\xi}_{j} \bar{\xi}_{k} \bar{\varphi}_{, j k}+O\left(a^{3}\right),
$$

since $\overline{\varphi^{\prime}}=0$ and $\bar{\xi}=0$. Evidently $\bar{\varphi}^{\mathrm{s}}$ is a wave property in the sense that it can be evaluated correct to leading order, in this case $O\left(a^{2}\right)$, from a knowledge of zero-order mean flow and linearized wave solutions only. For later reference we note another consequence of $(2.26)$, namely that

$$
\varphi^{l}=\varphi^{\prime}+\xi_{j} \bar{\varphi}_{, j}+O\left(a^{2}\right)
$$

recalling (2.17), (2.25), and the fact that $\bar{\varphi}^{S}=O\left(a^{2}\right)$.

The difference between the form of (2.21) and that of its Eulerian-mean counterpart

$$
\bar{D} \bar{S}+\bar{Q}=-\overline{\mathbf{u}^{\prime} \cdot \nabla S^{\prime}}
$$

is accounted for by the Stokes 'drift' $\overline{\mathbf{u}}^{\mathrm{S}}$ as well as the other Stokes corrections $\bar{S}^{\mathrm{s}}$ and $\bar{Q}^{\mathrm{s}}$. Whenever the term $-\overline{u^{\prime} \cdot \nabla S^{\prime}}$ in $(2.29)$ plays an essential role in forcing the Eulerian-mean flow, the Stokes drift tends to be correspondingly significant. For example it is essential even in the case of almost-plane inertio-gravity waves, for which $\overline{\mathrm{u}}^{\mathrm{S}}$ might at first sight appear negligible because the wave motion is approximately transverse. The contribution from $\overline{\mathrm{u}}^{\mathrm{s}}$ to the mean Coriolis force cannot be neglected in calculating the acceleration of the mean flow, and hence in calculating the effective transport of momentum by the waves (Grimshaw 1975; McIntyre 1977, 1978). 
The second term in (2.27) is usually negligible in problems of slowly varying, almost-plane waves, because $\bar{\varphi}$ is differentiated twice. Waveguides with sheared mean flows provide the commonest situations in which both terms of (2.27) are, by contrast, essential to leading order (when $\varphi$ is the velocity along the waveguide). Further aspects of the differences between the Eulerian-mean and generalized Lagrangianmean descriptions will emerge in $\S 8$.

\section{Basic theorems governing mean-flow evolution}

Our formalism has already exposed constraints on mean-flow evolution of the simple type (2.21). We now show that it leads to a similarly powerful way of expressing the equation of motion for the mean flow. The result in question, which like (2.21) is exact, contains as special cases the results of I and III connecting mean-flow evolution with wave dissipation, etc. It is closely related to the approximate equation (24) of Dewar (1970); it also contains Bretherton's form of Kelvin's circulation theorem, and indeed the details of Bretherton's analysis $(1971, \S 6.8)$ suggest the key initial step in the derivation to be presented here. That first step has been given explicitly by Soward [1972, equation (5.4)] in the slightly different context of a general transformation of the equation of motion. Soward's equation (5.4) involves the 'related velocity' $\mathbf{v}$ associated with a general transformation of the form (2.2); in the present context $\mathbf{v}$ becomes a mean quantity, $\overline{\mathbf{u}}^{\mathrm{L}}$, and hence the equation becomes one for mean-flow evolution, which takes the desired form after further manipulations involving the properties (2.7) and (2.10). It turns out that no anisotropic, wave-associated stress tensor appears anywhere in this equation, in striking contrast to the Eulerian-mean equation of motion with its Reynolds-stress term. The nonlinear forcing of the mean flow by the waves is expressed in terms of a vector wave property $\mathbf{p}$, whose $i$ th component is

$$
\mathrm{P}_{i}(\mathbf{x}, t)=-\overline{\xi_{j, i}\left\{u_{j}^{l}+(\boldsymbol{\Omega} \times \xi)_{j}\right\}},
$$

together with an irrotational, pressure-like contribution, a term in the form of a scalar times the mean entropy gradient, and terms explicitly involving dissipation or other departures from conservative motion. Here $\boldsymbol{\Omega}$ is the angular velocity of a rotating frame of reference. The vector $\mathbf{P}$ will be identified in the companion paper as the pseudomomentum per unit mass, and indeed the theorem about to be derived is related to the partial resemblance between the two symmetry operations underlying conservation of momentum on the one hand, and conservation of pseudomomentum on the other (Peierls 1976; Bretherton 1979; Andrews \& McIntyre 1978b). The minus sign in (3.1) is necessary in order that our general definition be consistent with the usual approximate formula (intrinsic wave-energy per unit mass) $\div$ (intrinsic phase velocity), as is obvious from (3.1) in a simple case such as $\boldsymbol{\Omega}=0, \mathbf{u}^{l} \doteq \mathbf{u}^{\prime} \propto \exp i k\left(x_{1}-c t\right)$.

Let the $j$ th component of the equation of motion for the total flow be

$$
D u_{j} / D t+2(\boldsymbol{\Omega} \times \mathbf{u})_{j}+\Phi_{, j}+\rho^{-1} p_{, j}+X_{j}=0,
$$

where the angular velocity $\boldsymbol{\Omega}$ is assumed constant and such that $\overline{\boldsymbol{\Omega}}=\boldsymbol{\Omega} ; \Phi=\Phi(\mathbf{x}, t)$ is a potential for the sum of the gravitational and centrifugal forces, $\rho$ is density, $p$ is pressure, and $\mathbf{X}=\mathbf{X}(\mathbf{x}, t)$ is a function which allows for any further contributions such as dissipative forces, and whose form is left completely general. We allow $\Phi$ to depend on $t$ as well as on $\mathbf{x}$ so that the astrophysically significant case of a self-gravitating 
fluid is included. Let $T(S, p)$ and $F(S, p)$ represent the thermodynamical dependence of temperature and density upon entropy $S$ per unit mass and pressure $p$. Let $H(S, p)$ similarly represent the enthalpy per unit mass; by definition it satisfies

and

$$
\partial H(S, p) / \partial p=1 / \rho=1 / F(S, p),
$$

$$
\partial H(S, p) / \partial S=T(S, p) .
$$

The corresponding internal energy per unit mass is

Let

$$
\varepsilon(S, p)=H(S, p)-p / F(S, p) .
$$

$$
q=-\left\{F\left(S^{\xi}, p^{\xi}\right)\right\}^{-1}+\left\{F\left(\bar{S}^{\mathrm{L}}, p^{\xi}\right)\right\}^{-1},
$$

an $O(a)$ wave property which in virtue of $(2.23)$ is zero for adiabatic motion. Moreover it may easily be seen that $\bar{q}$ is an $O\left(a^{2}\right)$ wave property, by Taylor-expanding (3.5) in $S^{\xi}=\bar{S}^{\mathbf{L}}+S^{l}$ and noting that $\overline{S^{l}}=\mathbf{0}$.

Following Soward (loc. cit.), we multiply (3.2), evaluated at the point $\mathbf{\Xi}=\mathbf{x}+\boldsymbol{\xi}$, by $\Xi_{j, i}$ and sum over $j$. Now

$$
\Xi_{j, i}\left(\Phi_{, j}\right)^{\xi}=\left(\Phi^{\xi}\right)_{, i}
$$

by $(2.4 b)$; and

$$
\begin{aligned}
\Xi_{j, i}\left(\frac{1}{\rho} p_{, j}\right)^{\xi} & =\frac{1}{\rho^{\xi}}\left(p^{\xi}\right)_{, i}=\left(p^{\xi}\right)_{, i}\left[\left\{F\left(\bar{S}^{\mathrm{L}}, p^{\xi}\right)\right\}^{-1}-q\right] \\
& =\left(p^{\xi}\right)_{, i} \partial H\left(\bar{S}^{\mathrm{L}}, p^{\xi}\right) / \partial p^{\xi}-\left(p^{\xi}\right)_{, i} q \\
& =\left\{H\left(\bar{S}^{\mathrm{L}}, p^{\xi}\right)\right\}_{, i}-\left(\bar{S}^{\mathrm{L}}\right)_{, i} T\left(\bar{S}^{\mathrm{L}}, p^{\xi}\right)-\left(p^{\xi}\right)_{, i} q .
\end{aligned}
$$

Thus the multiplication by $\Xi_{j, i}$ brings the pressure term $\left(\rho^{-1} p_{, j}\right)^{\xi}$ into the form of a pure gradient plus a baroclinic term involving the mean stratification $\left(\bar{S}^{\mathrm{L}}\right)_{, i}$ plus a term explicitly involving a dissipative or other departure from adiabatic motion. (This form is different from that of Soward, who did not distinguish in his equation (5.4) between buoyancy effects of adiabatic and of diabatic origin.) The remaining manipulations, which depend on (2.7), (2.10) and their consequences, are given in appendix $B$, and the final result is the following theorem:

Theorem I. Equation (3.2) implies that

$$
\begin{aligned}
\bar{D}^{\mathrm{L}}\left(\bar{u}_{i}^{\mathrm{L}}-\mathrm{P}_{i}\right)+\left(\bar{u}_{k}^{\mathrm{L}}\right)_{, i}\left(\bar{u}_{k}^{\mathrm{L}}-\mathrm{P}_{k}\right)+ & 2\left(\boldsymbol{\Omega} \times \overline{\mathbf{u}}^{\mathrm{L}}\right)_{i}+\Pi_{, i} \\
& -\left(\bar{S}^{\mathrm{L}}\right)_{, i} \overline{T\left(\bar{S}^{\mathrm{L}}, p^{\xi}\right)}+\bar{X}_{i}^{\mathrm{L}}=-\overline{\xi_{j, i} \bar{X}_{j}^{l}}+\overline{\left(p^{\xi}\right)_{, i} q},
\end{aligned}
$$

where

$$
\Pi \equiv \overline{H\left(\bar{S}^{\mathrm{L}}, p^{\xi}\right)}+\bar{\Phi}^{\mathrm{L}}-\overline{u_{j}^{\xi}\left\{\frac{1}{2} u_{j}^{\xi}+(\mathbf{\Omega} \times \xi)_{j}\right\}} \text {. }
$$

The right-hand side of $(3.8)$ is a wave property, since $\overline{\left(p^{\xi}\right)_{, i} q}=\overline{\left(p^{l}\right)_{, i} q}+\left(\bar{p}^{\mathrm{L}}\right)_{, i} \bar{q}$ and $\overline{p^{l}}=0$. It depends explicitly on disturbance quantities which represent departures from conservative motion. Two special cases important in connexion with the 'longitudinally symmetric' mean flows mentioned earlier are as follows.

Corollary I. If all mean quantities are independent of the Cartesian co-ordinate $x_{i}$, then

$$
\bar{D}^{\mathrm{L}}\left(\bar{u}_{i}^{\mathrm{L}}-\mathrm{p}_{i}\right)+2\left(\boldsymbol{\Omega} \times \overline{\mathbf{u}}^{\mathrm{L}}\right)_{i}+\bar{X}_{i}^{\mathrm{L}}=-\overline{\xi_{j, i} \bar{X}_{j}^{l}}+\overline{\left(p^{l}\right)_{, i} q} .
$$

In the last term, $p^{l}$ appears instead of $p^{\xi}$ because $\left(\bar{p}^{\mathrm{L}}\right)_{, i}=0$. 
Corollary II. If all mean quantities are axisymmetric about an axis through the origin of co-ordinates and parallel to $\Omega$, then by taking $\hat{\mathbf{z}} . \mathbf{x} \times(3.8)$, where $\hat{\mathbf{z}}$ is a unit vector such that $\boldsymbol{\Omega}=\Omega \hat{\mathbf{z}}$, we get $\dagger$

$$
\bar{D}^{\mathrm{L}}\left\{\hat{\mathbf{z}} \cdot \mathbf{x} \times\left(\overline{\mathbf{u}}^{\mathrm{L}}-\mathbf{p}\right)\right\}+2 \Omega \mathbf{x}^{\perp} \cdot \overline{\mathbf{u}}^{\mathrm{L}}+\hat{\mathbf{z}} \cdot \mathbf{x} \times \overline{\mathbf{X}}^{\mathrm{L}}=\epsilon_{i j k} \hat{\mathbf{z}}_{j} x_{k}\left\{-\overline{\xi_{m, i} X_{m}^{l}}+\overline{\left(p^{l}\right)_{, i} q}\right\}
$$

where $\epsilon_{i j k}$ is the alternating tensor, and

$$
\mathbf{x}^{\perp}=\mathbf{x}-(\hat{\mathbf{z}} \cdot \mathbf{x}) \hat{\mathbf{z}}
$$

the position vector relative to the nearest point on the axis.

Corollaries I and II are exact results corresponding to the small-amplitude, Eulerian-mean results given in papers I and III $(q . v$.$) . Despite being more general$ the present results are drastically simpler, as is especially evident by comparison with the formulae given in $\S 6$ of III. Corollaries I and II show how the longitudinal acceleration is linked to dissipation or forcing of the disturbances, a relation seldom obvious from an Eulerian-mean description. We shall examine this relation in more detail in $\S 5$.

It remains to demonstrate the connexion with Kelvin's and Bjerknes' circulation theorems (Eliassen \& Kleinschmidt 1957) and to note the special role of $p$ in irrotational flow.

Corollary III. Let $\Gamma$ be a closed circuit moving with velocity $\overline{\mathbf{u}}^{\mathrm{L}}$. Then

$$
\begin{aligned}
\frac{d}{d t} \oint_{\Gamma}\left(\overline{\mathbf{u}}^{\mathrm{L}}-\mathbf{p}+\mathbf{\Omega} \times \mathbf{x}\right) \cdot d \mathbf{s}-\oint_{\Gamma} \overline{T\left(\overline{\boldsymbol{S}}^{\mathrm{L}}, p^{\xi}\right)} d \bar{S}^{\mathrm{L}} & \\
& =-\oint_{\Gamma}\left\{\overline{X_{i}^{L}}+\overline{\xi_{j, i} X_{j}^{l}}-\overline{\left(p^{\xi}\right)_{, i} q}\right\} d s_{i} .
\end{aligned}
$$

This result follows immediately from theorem I and the standard identity

$$
\frac{d}{d t} \oint_{\Gamma} \varphi_{i} d s_{i}=\oint_{\Gamma}\left(\bar{D}^{\mathrm{L}} \varphi_{i}+\bar{u}_{k, i}^{\mathrm{L}} \varphi_{k}\right) d s_{i}
$$

in the present notation. It generalizes the approximate results obtained by Bretherton (1971) and Grimshaw (1975) for slowly varying waves. When $\mathrm{X}, q$ and $Q$ are zero it implies the version of Kelvin's circulation theorem given by Bretherton (1978), namely that the circulation of $\overline{\mathbf{u}}^{\mathrm{L}}-\mathbf{p}+\boldsymbol{\Omega} \times \mathbf{x}$ around any circuit lying in a surface of constant $\bar{S}^{L}$, and moving with the Lagrangian-mean flow, is exactly constant. This result is evidently related to (2.22) with $S$ replaced by Ertel's potential vorticity (see (5.15) below).

A deduction relevant to the classical theories of acoustic and surface gravity waves is the following:

CoRollary IV. If $\Omega=0$ and the motion is irrotational, homentropic ( $S=$ constant) and conservative $(\mathbf{X}=0, q=0)$, then $\overline{\mathbf{u}}^{\mathrm{L}}-\mathbf{p}$ is irrotational.

This follows immediately from (3.11) and postulate (viii), since irrotational, conservative flow can be set up from an initial state of rest. (An alternative, more direct proof due to Soward will be noted in $\$ 7$.)

$\dagger$ In deriving (3.10) we have used the fact that axisymmetry of the mean flow implies that $\hat{\mathbf{z}} \cdot \mathbf{x} \times \nabla \bar{\varphi}=0$ if $\varphi$ is a scalar, and $\hat{\mathbf{z}} \cdot \mathbf{x} \times \nabla \bar{\varphi}-\hat{\mathbf{z}} \times \bar{\varphi}=0$ if $\varphi$ is a vector. (The latter relation implies that the extra term produced by taking $\hat{\mathbf{z}} \cdot \mathbf{x} \times$ inside $\bar{D}^{\mathrm{L}}$ is cancelled by the term in $\left(\bar{u}_{k}^{\mathrm{I}}\right)_{, i} \mathbf{P}_{k}$.) If it is desired to express the equations in cylindrical or spherical co-ordinates the standard formulae may be used for each tensor field, including $\xi(x, t)$. This implies of course that $\xi$ is resolved into its components on the co-ordinate directions at $\mathbf{x}$. and not those at $\mathbf{x}+\xi$. 
In corollary III we may note an alternative form of the Bjerknes buoyancy term on the left:

$$
-\oint_{\Gamma} \overline{T\left(\bar{S}^{\mathrm{L}}, p^{\bar{\xi}}\right)} d \bar{S}^{\mathrm{L}}=\oint_{\Gamma} \overline{d p^{\xi} / F\left(\bar{S}^{\mathrm{L}}, p^{\bar{\xi}}\right)}
$$

which follows because $d H=T d S+\rho^{-1} d p, \overline{()} d \bar{S}^{\mathrm{L}}=\overline{() d \bar{S}^{\mathrm{L}}}$, and $\overline{d H}=d(\bar{H})$ by postulate (iv). (This can be combined with the $q$ term on the right of (3.11), noting (3.5), to give $\oint(\overline{(d p / \rho)})^{\mathrm{L}}$ on the left.)

If we were to extend the meaning of the operator ( ) so as to be applicable to a line integral $I$ over a path $\Gamma$, then we could rederive (3.11), in the conservative case, direct from Bjerknes' circulation theorem for the total flow just as in Bretherton (1979). (For instance when $\left(\right.$ ) denotes averaging with respect to $x_{1}, I$ would be defined as the average of integrals over the set of paths similar to $\Gamma$ obtained by translating $\Gamma$ in the $x_{1}$ direction.) One would then add to the list of axioms one which asserts that ( ), so extended, commutes with line integration. But it is of some interest that our result (3.11) does not actually depend on any such extension of the meaning of $\overline{(}$ ).

We end this section on basic theorems with a result which bears the same relation to the energy equation as theorem I does to the equation of motion itself:

TheOREM II. Equation (3.2) implies that

$$
\begin{aligned}
\bar{D}^{\mathrm{L}}\left(\frac{1}{2} \sqrt{\left.\mathbf{u}^{\xi}\right|^{2}}+\bar{H}^{\mathrm{L}}+\bar{\Phi}^{\mathrm{L}}-\mathrm{e}\right)-\left(\bar{u}_{k}^{\mathrm{L}}\right)_{, t}\left(\bar{u}_{k}^{\mathrm{L}}-\mathrm{P}_{k}\right)-\Pi_{, t} \\
+\left(\bar{S}^{\mathrm{L}}\right)_{, t} \overline{T\left(\bar{S}^{\mathrm{L}}, p^{\xi}\right)}+\overline{\mathbf{u}}^{\mathrm{L}} \cdot \overline{\mathbf{X}}^{\mathrm{L}}+(\overline{T Q})^{\mathrm{L}}=-\bar{u}_{k}^{\mathrm{L}} \overline{\xi_{j, k} \bar{X}_{j}^{l}}-\overline{\left(p^{\xi}\right)_{, t} q},
\end{aligned}
$$

where the Lagrangian-mean enthalpy $\left.\bar{H}^{\mathrm{L}}=\overline{H(S, p)^{\mathrm{L}}}=\overline{H\left(\mathcal{S}^{\xi}, p^{\xi}\right.}\right)$, and

$$
\mathrm{e}=\overline{\xi_{j, t}\left\{u_{j}^{l}+(\boldsymbol{\Omega} \times \xi)_{j}\right\}},
$$

i.e. minus the temporal analogue of $(3.1)$ (see $\$ 5$ of the companion paper). (Note the result corresponding to corollary $I$ when mean quantities are independent of time.) The derivation, omitted here, parallels that of theorem I (see appendix B). There, the momentum equation (3.2) was multiplied by $\Xi_{j, i}$. Here, we contract the four-vector $-\left(\Xi_{1}, \Xi_{2}, \Xi_{3},-t\right)_{, t}$ with the set of four equations made up of the three components of the momentum equation together with the energy equation corresponding to (3.2), namely

$$
D\left(\frac{1}{2}|\mathbf{u}|^{2}+\varepsilon+\Phi\right) / D t+\rho^{-1} \nabla \cdot(p \mathbf{u})=-\mathbf{u} \cdot \mathbf{X}-T Q+\Phi_{, t},
$$

where $\varepsilon$ is given by (3.4). In proving (3.13) it is convenient first to replace $\rho^{-1} \nabla \cdot(p \mathbf{u})$ in (3.15) by $D\left(p \rho^{-1}\right) / D t-\rho^{-1} p_{, t}$ using (4.1), and then to recall (3.6), (3.7) and (2.4).

\section{Mass conservation}

To complete the set of equations for the mean flow, and to express them in conservation form when appropriate, we need a mass continuity equation. The required analysis also provides the easiest way of deducing the boundary condition satisfied by $\overline{\mathbf{u}}^{\mathrm{L}}$ at a surface generating, reflecting or absorbing a wave disturbance. Moreover it leads, in the case where $(\overrightarrow{)}$ is a Cartesian space average, to a particularly simple way of visualizing $\overline{\mathbf{u}}^{\mathrm{L}}$, as the velocity of the centre of mass of a tube of fluid initially lying parallel to the direction of averaging. This appears as a consequence of another key property of the GLM description, namely that the total momentum is on average attributable to the mean flow, with no contribution from the waves. 


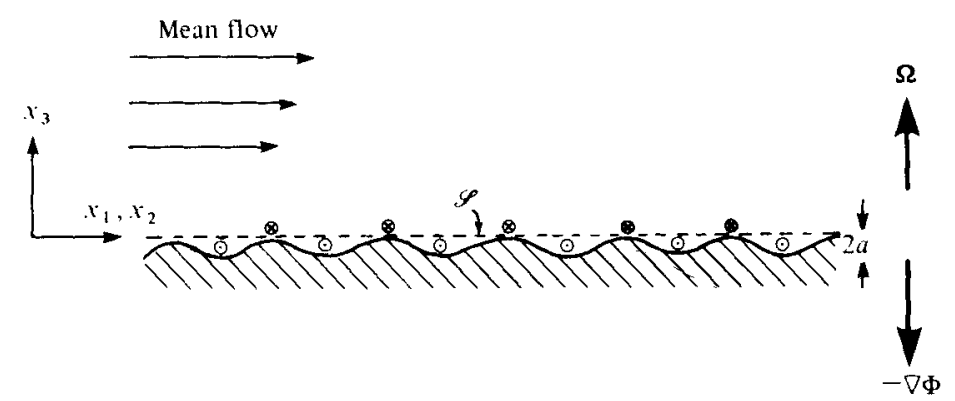

Figure 2. Generation of inertio-gravity waves by flow of a stratified, rotating fluid over a corrugated, impermeable boundary. The disturbance velocity out of the paper tends to be in phase with the depressions in the boundary, leading to an $O\left(a^{2}\right)$ mass flux beneath the plane $\mathscr{P}$. (The signs shown for the disturbance velocity are appropriate to the case where $2 \Omega$ is less than the buoyancy frequency of the stratification.)

\subsection{The Jacobian J and the mean-flou density $\tilde{\rho}$}

We now assume the following, this being the last in our list of postulates:

(ix) The total motion $\mathbf{u}(\mathbf{x}, t)$ conserves mass, with density $\rho$ :

$$
D \rho / D t+\rho \nabla \cdot \mathbf{u}=0 .
$$

It is convenient to associate with the mean velocity field $\overline{\mathbf{u}}^{\mathrm{L}}(\mathbf{x}, t)$ a density $\tilde{\rho}$ defined such that the mean flow also satisfies a mass-conservation equation exactly:

$$
\bar{D}^{\mathrm{L}} \tilde{\rho}+\tilde{\rho} \nabla \cdot \overline{\mathbf{u}}^{\mathrm{L}}=0 .
$$

The required definition is (Eckart 1963)

$$
\tilde{\rho}=\rho^{\xi} J
$$

where $J$ is the Jacobian of the mapping $\mathbf{x} \rightarrow \mathbf{x}+\xi \equiv \mathbf{E}(\mathbf{x}, t)$ :

$$
J=\operatorname{det}\left\{\boldsymbol{\Xi}_{i, j}\right\}=\operatorname{det}\left\{\delta_{i j}+\xi_{i, j}\right\} .
$$

By postulate (vii), $J$, and therefore $\tilde{\rho}$, is finite and positive. A number of standard analytical properties of $J$ and its cofactors $K_{i j}$ will be needed later; these are listed in appendix $A$ for convenience. To show that (4.3) satisfies (4.2) note that, by (4.3) and the rule for changing variables of integration,

$$
\int_{\mathscr{\mathscr { C }}} \tilde{\rho}(\mathbf{x}, t) d \mathbf{x}
$$

is equal to the mass instantaneously contained in a volume $\mathscr{V}^{\mathscr{E}}$ which is the image of $\mathscr{V}$ under the mapping $x \rightarrow \Xi$. If $\mathscr{V}^{\sim}$ moves with the mean flow then $\mathscr{V}^{\xi}$ moves with the actual fluid, by $(2.10 a)$, and (4.5) is constant. This is true for arbitrary $\mathscr{V}$, whence $(4,2)$ follows by a standard argument. An alternative, direct proof is given in appendix A.

It is crucial to subsequent developments that

$$
\overline{\tilde{\rho}}=\tilde{\rho},
$$

i. $\Theta$. that $\tilde{\rho}$ is a mean quantity. This is a consequence of postulate (viii), and the constancy of (4.5) for a small volume $\mathscr{V}^{r}$ which follows the set of moan trajectories passing through an arbitrary neighbourhood of the 'undisturbed' point $\mathbf{x}_{\mathbf{0}}$ at time $t_{0}$. Again, an alternative and more direct proof is given in appendix $A$. 


\subsection{Condition at an impermeable boundary}

If the flow is constrained by an impermeable boundary $\mathscr{B}^{\xi}$, such as the corrugated boundary shown in figure 2 , then the corresponding 'mean boundary' $\mathscr{B}$, defined as the image of $\mathscr{B}^{\xi}$ under the inverse mapping $\mathbf{\Xi} \rightarrow \mathbf{x}$, is impermeable to the Lagrangianmean flow. This is clear from the foregoing considerations of mass conservation, noting that $\mathscr{B}^{\xi}$ may be regarded as 'moving with the actual fluid' for present purposes. Thus the boundary condition on the mean flow is that the component of $\overline{\mathbf{u}}^{\mathrm{L}}$ normal to the 'mean boundary' $\mathscr{B}$ equals the normal component of the velocity of $\mathscr{B}$ itself.

It should be appreciated that a correspondingly simple statement is far from true in an Eulerian-mean description as is, indeed, implied by the differences between Eulerian- and Lagrangian-mean descriptions already discussed in $\S 2.4$. The normal component of the Eulerian-mean velocity generally disagrees with that of the boundary. One very simple example involving acoustic waves is given in $\S 6$. Another example is that of figure 2 , which depicts a stratified, rotating flow over a rigid, corrugated lower boundary, generating inertio-gravity waves. (More details for this example are given in McIntyre 1977, 1978.) If the boundary is immobile, then the Lagrangian-mean vertical velocity $\bar{u}_{3}^{\mathrm{L}}$ is zero there. There can however be a significant Eulerian-mean velocity component $\bar{u}_{3}$, generally needed to satisfy continuity with the horizontal mass flux below an Eulerian control surface such as $\mathscr{S}$ in the figure (Uryu 1974; Bretherton \& Haidvogel 1976). The mass flux in turn arises from a correlation between the boundary height and the disturbance velocity normal to the paper. This difference between the Eulerian- and Lagrangian-mean boundary conditions is of course just that required to accommodate the Stokes drift (whose normal component is seldom zero at a boundary generating waves).

It thus appears that the generalized Lagrangian mean is a natural way of defining 'mean flow' when simple boundary conditions on the normal component of mean velocity are desired.

\section{3. $\overline{\mathbf{u}}^{\mathrm{L}}$ as the motion of the centre of mass of a tube of fluid}

The total momentum in the volume $\mathscr{V}^{\mathcal{E}}$ is

By (4.3) this is $\int \tilde{\rho} \mathbf{u}^{\xi} d \mathbf{x}$. Thus

$$
\int_{y-\xi} \rho \mathbf{u} d \mathbf{x}=\int_{\mathbf{x} \in \mathscr{Y}^{-}} \rho^{\xi} \mathbf{u}^{\xi} d \mathbf{\Xi}(\mathbf{x})
$$

$$
\int_{\mathscr{r} \boldsymbol{\xi}} \rho \mathbf{u} d \mathbf{x}=\int_{\mathscr{V}^{-}} \tilde{\rho} \overline{\mathbf{u}}^{\mathbf{L}} d \mathbf{x}+\int_{\mathscr{\mathscr { V }}} \tilde{\rho} \mathbf{u}^{l} d \mathbf{x} .
$$

Now recall the situation shown in figure $1(b)$, for the case in which ( ) is an average in the $x_{1}$ direction. Take $\mathscr{Y}$ to be a long, thin, straight, uniform tube of fluid of length $2 b$, extending between $x_{1}= \pm b$. Then (4.6) and (4.7) imply that

$$
\lim _{b \rightarrow \infty} \frac{1}{2 b} \int_{\mathscr{y}-\boldsymbol{\xi}} \rho \mathbf{u} d \mathbf{x}=\lim _{b \rightarrow \infty} \frac{1}{2 b} \int_{\mathscr{\gamma}} \tilde{\rho} \overline{\mathbf{u}}^{\mathrm{L}} d \mathbf{x},
$$

since $\overline{\mathbf{u}^{l}}=0$, so that the last integral in (4.7) is irrelevant. If $\mathscr{V}$ always moves with velocity $\overline{\mathrm{u}}^{\mathrm{L}}$, just as does the rigid rod $R$ in figure $1(a)$, then $\mathscr{V}^{-\xi}$ is the actual tube of fluid which initially lay along $R$. By (4.5) and (4.8), its momentum per unit length is 
equal, in the limit, to that of the same mass of fluid moving rigidly with velocity $\overline{\mathbf{u}}^{\mathrm{L}}$. (In this sense all the mean momentum is contained in the mean flow; the waves possess no momentum on average.) It follows that $\overline{\mathbf{u}}^{\mathbf{L}}$ is the velocity of the centre of mass of $\mathscr{V}^{\xi}$.

\section{The mean-flow equations and some of their general implications}

\subsection{A complete set of equations}

We now have the equation of motion in the form (3.8), the continuity equation (4.2), and an equation in the form (2.21) for the mean entropy. These constitute five equations for the six mean quantities

$$
\left\{\overline{\mathbf{u}}^{\mathrm{L}}, \overline{\boldsymbol{S}}^{\mathrm{L}}, \bar{p}^{\mathrm{L}}, \tilde{\rho}\right\},
$$

so one more equation is needed. Applying ()$^{\mathrm{L}}$ to the equation of state in the form

we rearrange the result as

$$
\rho-F(S, p)=0 \text {, }
$$

$$
\tilde{\rho}-F\left(\bar{S}^{\mathrm{L}}, \bar{p}^{\mathrm{L}}\right)=\Delta,
$$

where $\Delta$ is an $O\left(a^{2}\right)$ wave property:

$$
\Delta=\left(\tilde{\rho}-\bar{\rho}^{\mathrm{L}}\right)+\overline{F\left(S^{\xi}, p^{\xi}\right)}-F\left(\bar{S}^{\mathrm{L}}, \bar{p}^{\mathrm{L}}\right) .
$$

The equation of state also enters (3.8) via the known functions $T(S, p)$ and $H(S, p)$. A form of the equation of motion which does not involve $T$ and $H$ will be given in $\S 8.1$.

The self-gravitating case may be treated simply by appending the appropriate Poisson equation relating $\Phi$ to $\rho$; its Eulerian mean appears to be the most convenient form to use.

\subsection{Longitudinally symmetric mean flow}

We now apply this set of equations to a qualitative discussion of mean-flow evolution, with emphasis on the astrogeophysical type of problem involving stable stratification and Coriolis forces and in which the mean flows of interest are axisymmetric. At sufficiently large distances from the symmetry axis we have the limiting case appropriate to corollary I [equation (3.9)], in which the azimuthal or longitudinal co-ordinate, of which mean quantities are assumed independent, can be treated as a Cartesian co-ordinate $x_{1}$. We shall adopt that case as the simplest basis for detailed discussion. However, by virtue of corollary II [equation (3.10)] the discussion applies almost word for word to longitudinally symmetric mean flows in general.

In Cartesian components the complete set of equations for the rectilinear limiting case, with $\Omega=(0,0, \Omega)$ and $\bar{\Phi}=\bar{\Phi}\left(x_{2}, x_{3}\right)$ or $\bar{\Phi}\left(x_{2}, x_{3}, t\right)$, may be written

$$
\begin{gathered}
\left(\bar{u}_{1}^{\mathrm{L}}-\mathrm{P}_{1}\right)_{, t}+\left(\bar{u}_{1,2}^{\mathrm{L}}-\mathrm{P}_{1,2}-2 \Omega\right) \bar{u}_{2}^{\mathrm{L}}+\left(\bar{u}_{1,3}^{\mathrm{L}}-\mathrm{P}_{1,3}\right) \bar{u}_{3}^{\mathrm{L}}=-\mathscr{X}_{1}, \\
\bar{u}_{2, t}^{\mathrm{L}}+\bar{u}_{2}^{\mathrm{L}} \bar{u}_{2,2}^{\mathrm{L}}+\bar{u}_{3}^{\mathrm{L}} \bar{u}_{2,3}^{\mathrm{L}}+2 \Omega \bar{u}_{1}^{\mathrm{L}}-\bar{S}_{, 2}^{\mathrm{L}} T\left(\bar{S}^{\mathrm{L}}, \bar{p}^{\mathrm{L}}\right)+\pi_{, 2}=-\mathscr{X}_{2}, \\
\bar{u}_{3, t}^{\mathrm{L}}+\bar{u}_{2}^{\mathrm{L}} \bar{u}_{3,2}^{\mathrm{L}}+\bar{u}_{3}^{\mathrm{L}} \bar{u}_{3,3}^{\mathrm{L}}-\bar{S}_{, 3}^{\mathrm{L}} T\left(\bar{S}^{\mathrm{L}}, \bar{p}^{\mathrm{L}}\right)+\pi_{, 3}=-\mathscr{X}_{3}, \\
\bar{S}_{, t}^{\mathrm{L}}+\bar{u}_{2}^{\mathrm{L}} \bar{S}_{, 2}^{\mathrm{L}}+\bar{u}_{3}^{\mathrm{L}} \bar{S}_{, 3}^{\mathrm{L}}=-\bar{Q}^{\mathrm{L}}, \\
\tilde{\rho}-F\left(\bar{S}^{\mathrm{L}}, \bar{p}^{\mathrm{L}}\right)=\Delta \\
\tilde{\rho}_{, t}+\left(\tilde{\rho} \bar{u}_{2}^{\mathrm{L}}\right)_{, 2}+\left(\tilde{\rho} \bar{u}_{3}^{\mathrm{L}}\right)_{, 3}=0
\end{gathered}
$$

where

$$
\pi=H\left(\bar{S}^{\mathrm{L}}, \bar{p}^{\mathrm{L}}\right)+\bar{\Phi}
$$


and where the Poisson equation for $\bar{\Phi}$ is appended if appropriate. In (5.5a), $\mathscr{X}_{1}$ is given by (3.9) (corollary I) as

$$
\mathscr{X}_{1}=\bar{X}_{1}^{\mathrm{L}}+\overline{\xi_{j, 1} \bar{X}_{j}^{l}}-\overline{\left(p^{l}\right)_{, 1} q}
$$

while $\mathscr{X}_{2}$ and $\mathscr{X}_{3}$ are more complicated expressions with whose details we need not be concerned except for the fact that, like $\mathscr{X}_{1}$, they are wave properties apart from a possible contribution from $\overline{\mathbf{X}}^{\mathrm{L}} \cdot \dagger$

We first ask under what circumstances a steady, unidirectional mean flow, with

and

$$
\partial\left\{\bar{u}_{1}^{\mathrm{L}}, \bar{u}_{2}^{\mathrm{L}}, \bar{u}_{3}^{\mathrm{L}}, \bar{p}^{\mathrm{L}}, \tilde{\rho}, \bar{S}^{\mathrm{L}}, \bar{\Phi}\right\} / \partial t=0
$$

$$
\left\{\bar{u}_{1}^{\mathrm{L}}, \bar{u}_{2}^{\mathrm{L}}, \bar{u}_{3}^{\mathrm{L}}\right\}=\left\{\bar{u}_{1}^{\mathrm{L}}, 0,0\right\}
$$

is allowed by the equations in the presence of waves. In other words, when is the forcing on the right of (5.5) not such as to cause a departure from a steady, unidirectional mean flow of the form (5.7)? We assume of course that this flow is permitted by the boundary conditions. The answer is evident from inspection of (5.5): the steady mean flow (5.7) is possible only if

and

$$
\mathscr{X}_{1}=\bar{Q}^{\mathrm{L}}=0
$$

$$
\partial\left\{\mathrm{p}_{1}, \mathscr{X}_{2}, \mathscr{X}_{3}, \Delta\right\} / \partial t=0
$$

which by (5.6) generally requires the waves to be not only steady, but also conservative. This important fact could hardly have been deduced by inspection of the Eulerianmean equations (e.g. III, equations 6.2).

It should be particularly noted that $\mathscr{X}_{2}, \mathscr{X}_{3}$ and $\Delta$ are not generally required to be zero. For instance they need not be zero for stable, stratified rotating flows in which $\nabla \bar{\Phi}, \nabla \bar{S}^{\mathrm{L}}$ and $\boldsymbol{\Omega}$ are non-zero. In such flows the $\overline{\mathbf{u}}^{\mathrm{L}}, \bar{S}^{\mathrm{L}}$ and $\bar{p}^{\mathrm{L}}$ fields can adjust so that Coriolis, buoyancy and pressure forces balance $\mathscr{X}_{2}$ and $\mathscr{X}_{3}$ : the resulting steady, unidirectional mean flow must satisfy the equation

$$
-2 \Omega . \nabla \bar{u}_{1}^{\mathrm{L}}+\left\{\nabla \bar{S}^{\mathrm{L}} \times \nabla T\left(\bar{S}^{\mathrm{L}}, \bar{p}^{\mathrm{L}}\right)\right\}_{1}=-\mathscr{X}_{3,2}+\mathscr{X}_{2,3},
$$

which represents a modified 'thermal wind' or geostrophic-hydrostatic balance and which results from cross-differentiating $(5.5 b)$ and $(5.5 c)$, setting $\bar{u}_{2}^{\mathrm{L}}=\bar{u}_{3}^{\mathrm{L}}=0$. In such circumstances the interaction of the waves and mean flow, while by no means zero, as (5.9) illustrates, is not such as to force the mean flow to accelerate.

If on the other hand $\mathscr{X}_{1}$ or $\bar{Q}^{\mathrm{L}}$ differ from zero, for steady or approximately steady waves, then cumulative mean-flow changes must be expected to occur as time goes on, essentially because there is no longitudinal mean pressure gradient in $(5.5 a)$ which can balance the effects of $\mathscr{X}_{1}$ and $\bar{Q}^{\mathrm{L}} . \ddagger$

$\dagger$ Explicitly,

$$
\begin{aligned}
& \mathscr{P}_{i}=\bar{X}_{i}^{\mathrm{L}}+\overline{\xi_{j, i} X_{j}^{l}}-\overline{\left(p^{\xi}\right)_{, i} q}-\bar{D}^{\mathrm{L}} \mathbf{p}_{i}-\left(\bar{u}_{k}^{\mathrm{L}}\right)_{, i} \mathbf{p}_{k} \\
& \left.-\bar{S}_{. i}^{\mathrm{L}} \overline{\left(T\left(\overline{\bar{S}^{\mathrm{L}}, p^{\xi}}\right)\right.}-T\left(\bar{S}^{\mathrm{L}}, \bar{p}^{\mathrm{L}}\right)\right\}+\left[\overline{H\left(\bar{S}^{\mathrm{L}}, p^{\bar{\zeta}}\right)}-H\left(\bar{S}^{\mathrm{L}}, \bar{p}^{\mathrm{L}}\right)+\bar{\Phi}^{\mathrm{s}}-u_{j}^{l\left(\frac{1}{2} u_{j}^{l}+(\bar{\Omega} \times \xi)\right\}}\right]_{, i}
\end{aligned}
$$

for $i=2$ or 3, this being just the obvious result of rearranging terms in (3.8). Simpler-looking alternative versions of $(5.5 b, c)$ will be noted in $\$ 8$.

$\ddagger \bar{Q}^{L}$ of course acts indirectly, by inducing a mean secondary circulation $\left(\bar{u}_{2}^{L}, \bar{u}_{3}^{L}\right)$ in the familiar way illustrated for instance in $I$, in MoIntyre $(1977,1978)$, and in $\S 9$ below. 


\subsection{Transient, conservative waves}

When the motion is conservative, the restrictions on mean-flow change are severe even if the waves are not steady. Then $(5.5 a)$ becomes

and $(5.5 d)$ becomes

$$
\bar{D}^{\mathrm{L}}\left(\bar{u}_{1}^{\mathrm{L}}-\mathrm{p}_{1}-2 \Omega x_{2}\right)=0
$$

$$
\bar{D}^{\mathrm{L}} \bar{S}^{\mathrm{L}}=0 \text {. }
$$

Taken together, this pair of constraints may be regarded as the extension to finite amplitude of the 'generalized Charney-Drazin theorem' for transient waves, a linear version of which was presented and discussed in $\S 5$ of $\mathrm{I}$. (By (3.10) the analogue of $(5.10 a)$ for the more general, axisymmetric case is simply

$$
\bar{D}^{\mathrm{L}}\left\{x^{\perp}\left(\bar{u}_{1}^{\mathrm{L}}-\mathrm{P}_{1}\right)+\Omega x^{\perp 2}\right\}=0,
$$

where $\bar{u}_{1}^{\mathrm{L}}$ is the longitudinal mean velocity component, and $x^{\perp}$ is the perpendicular distance to the axis.)

Under at least some circumstances of interest the results (5.10) appear to imply that a temporary disturbance can force only a temporary mean-flow change, even at finite amplitude. Suppose that we have a domain $\mathscr{D}$, in the $x_{2}, x_{3}$ plane, initially containing a steady, undisturbed flow of the type (5.7), through which a nonlinear disturbance subsequently propagates. We suppose that no disturbance is left behind, in the sense that $\mathbf{p}=0$ for $t<t_{0}, t>t_{f}$,

say, and similarly for the right-hand sides of (5.5). The disturbance might for example be generated at one part of the boundary of $\mathscr{D}$ and absorbed (conservatively) at another, or alternatively the domain might be unbounded. Then after time $t_{f}$ the mean flow is left as before apart from longitudinally symmetric oscillations (described by the unforced mean equations) about the original steady state; we here assume that the flow satisfies the classical criterion for stability to longitudinally symmetric disturbances (e.g. Charney 1973; Hoskins 1974), as is normally true for the astrogeophysical flows of interest. If the symmetric oscillations in turn propagate away, or are absorbed conservatively at a boundary, the mean flow reverts exactly to its original state whenever the information given by (5.10) for $t>t_{f}$, i.e. prescribed values of $\bar{u}_{1}^{\mathrm{L}}-2 \Omega x_{2}$ and $\bar{S}^{\mathrm{L}}$ on each line of fluid particles parallel to $x_{1}$, is enough to determine uniquely an undisturbed flow of the type (5.7) satisfying (5.9) with righthand side zero. This in turn can be expected to be the case whenover both $(a)$ the boundary conditions allow no fluid to enter or leave $\mathscr{D}$ permanently (as if $\mathscr{D}$ were a rigid container, at least so far as the final state is concerned), and $(b)$ the flow for $t>t_{f}$ is stable to longitudinally symmetric disturbances as already assumed. (Note that stability in this sense requires $\bar{S}^{\mathrm{L}}\left(x_{2}, x_{3}\right)$ to increase monotonically upwards, i.e. in the direction of decreasing $p$, and $\bar{u}_{1}^{\mathrm{L}}\left(x_{2}, x_{3}\right)-2 \Omega x_{2}$ to increase monotonically along each constant $\bar{S}^{\mathrm{L}}$ surface in the direction consistent with centrifugal stability; this would appear to permit only one arrangement of the fluid elements in the $x_{2}, x_{3}$ plane for simple shapes of $\mathscr{D}$, but surprisingly we have not been able to find a uniqueness proof in the literature.) $\dagger$

+ The mathematical statement of the problem involves a Monge-Ampère equation. A proof of uniqueness has been given by Rellich (see Courant \& Hilbert 1962, p. 324) but under physically inappropriate boundary conditions.

Note added in proof. In the case of a Boussinesq, incompressible fluid, and convex $\mathscr{D}$, a suitable uniqueness theorem is given by A. V. Pogorelov (Monge-Ampère Equations of Elliptic Type, p. 56, theorem 2, Noordhoff, 1964). 
If we are given only that

$$
\mathbf{u}^{\prime}=p^{\prime}=\rho^{\prime}=S^{\prime}=0 \text { for } t<t_{0} \text { and } t>t_{f},
$$

then it is not in fact obvious whether (5.12) will hold, i.e. whether $\mathbf{p}$ will return to zero for $t>t_{f}$, since in (3.1), $\xi$ and thus $\mathbf{u}^{l}$ might not return to zero even though (5.13) was satisfied. But it can be shown that a sufficient condition on conservative flow, guaranteeing that $\xi$ and therefore $p$ does vanish whenever and wherever $(5.13)$ is satisfied, is that the Jacobian

for all $x_{2}, x_{3}$, where

$$
\partial\left(\bar{S}^{\mathrm{L}}, \bar{Z}^{\mathrm{L}}\right) / \partial\left(x_{2}, x_{3}\right) \neq 0
$$

$$
Z \equiv \rho^{-1}(2 \Omega+\nabla \times \mathbf{u}) \cdot \nabla S
$$

i.e. Ertel's potential vorticity. (Or $Z$ can be any other quantity satisfying $D Z / D t=0$ and hence $\bar{D}^{L} \bar{Z}^{L}=0$.) The proof, which relies on the basic property $\bar{\xi}=0$ expressed by (2.7), is given in appendix $\mathrm{C}$. Note that the relation $\bar{D}^{\mathrm{L}} \bar{Z}^{\mathrm{L}}=0$ together with $(5.10 b)$ and (4.2) or (5.5f) implies that

$$
\bar{D}^{\mathrm{L}}\left\{\tilde{\rho}^{-1} \partial\left(\bar{S}^{\mathrm{L}}, \bar{Z}^{\mathrm{L}}\right) / \partial\left(x_{2}, x_{3}\right)\right\}=0
$$

(expressing proportionality of the area of a 'mean fluid element' to $\tilde{\rho}^{-1}$ ), so that (5.14) is true for all $t$ if true initially. It is possible that a condition weaker than (5.14) would suffice, but we have not succeeded in finding a general proof in any such case.

The requirement that the flow be conservative may be stringent in practice. If a very small amount of dissipation is present, its effects can interact strongly with transient mean-flow changes that would otherwise be temporary (see for instance examples studied by Geisler 1974, Holton \& Mass 1976, and Holton \& Dunkerton 1978, involving stratospheric planetary waves). If the conditions for 'singular absorption' are approached somewhere (small phase velocity relative to the local mean flow, in the case of planetary waves), as a result of mean-flow changes due in the first instance to wave transience, then the dissipation terms may become more effective than an $a$ priori estimate would suggest.

\subsection{Resonant forcing of the mean flow}

If $\bar{u}_{2}^{\mathrm{I}}$ or $\bar{u}_{3}^{\mathrm{L}}$ is substantially different from zero, there may arise the possibility of resonant forcing of the mean flow (by terms like $\overline{\mathbf{u}}^{\mathbf{L}} . \nabla \mathbf{p}$ ). There can then be cumulative mean-flow changes, even for steady, conservative waves, when disturbance parameters are 'tuned' to special values. This possibility is in fact more familiar for mean flows which are not longitudinally symmetric. The simplest examples involve non-dispersive waves in one dimension, for which the 'tuning' is automatic because of the lack of dispersion. Another very simple example is that of a packet of internal gravity waves guided between fixed, horizontal surfaces, in a layer of inviscid, non-diffusive, Boussinesq liquid of constant mean buoyancy frequency $N$. We denote the mode number of the vertical waveguide structure by $n$. The waves have an amplitude which varies slowly in the propagation direction, so that we may define $(\overline{)}$ in the usual way to mean 'take the slowly-varying part of ( )'. When the waves have constant dimensionless frequency

$$
\omega / N=0.608
$$


(McIntyre 1973) then $O\left(a^{2}\right)$ mean-flow changes, which have the structure of very long waves with vertical mode number $2 n$, propagate at a phase velocity just matching the group velocity $c_{\mathrm{g}}$ of the primary waves. The result is resonant growth of the $O\left(a^{2}\right)$ mean flow. Thus in a frame of reference moving with velocity $c_{\mathrm{g}}$ we have an $O(a)$ wave amplitude which is steady to leading order, but cumulative mean-flow change. (See McIntyre (1973) for more detail, and Grimshaw (1976) for an analysis dealing with events after the simplest approximation scheme breaks down.) Watson, West \& Cohen (1976) and McComas \& Bretherton (1977) point out that this kind of resonant forcing can be viewed as a limiting case of resonant-triad interactions (contrary to an illconsidered statement by McIntyre!) and give other examples of it, involving both surface and internal waves, which may be important in the ocean.

\section{On irrotational waves}

The importance of departures from conservative wave motion, or of the tuning of phase and group velocities to give resonantly-forced mean-flow changes, should not distract attention from the fact that non-trivial mean flows can arise even in very simple examples of steady, conservative waves. This is well illustrated by the example from acoustics shown in figure 3, namely the steady, inviscid streaming associated with radiation from a rigid sphere oscillating in a straight line. The example (which surprisingly does not seem to be well known) also reinforces the point about the importance of boundary conditions made in $\$ 4.2$, as we shall see.

The total velocity $\mathbf{u}(\mathbf{x} \cdot t)$ is irrotational, so that

$$
\nabla \times\left(\overline{\mathbf{u}}^{\mathrm{L}}-\mathbf{p}\right)=0
$$

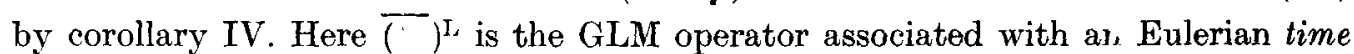
average ( ). There is however no reason for expecting that $\overline{\mathbf{u}}^{\mathrm{L}}=\mathbf{p}$ [even though the latter is true for certain very simple, and often quoted, solutions describing surfacegravity and acoustic waves, e.g. Landau \& Lifshitz 1959, equation (64.8)]. $†$ In this problem we have

$$
\overline{\mathbf{u}}^{\mathrm{L}} \cdot \hat{\mathbf{n}}=0
$$

at the mean position of the boundary $(\S 4.2)$, but $\mathbf{p} . \hat{\mathbf{n}} \neq 0$, where $\hat{\mathbf{n}}$ is a unit vector normal to the mean boundary. Assuming a steady wave field so that $\tilde{\rho}_{, t}=0$ in (4.2), we also have

$$
\nabla \cdot\left(\tilde{\rho} \overline{\mathbf{u}}^{\mathrm{L}}\right)=0 .
$$

Equations (6.1)-(6.3) govern $\overline{\mathbf{u}}^{\mathrm{L}}$ exactly.

For a simple analytical solution of $(6.1)-(6.3)$ correct to $O\left(a^{2}\right)$ we can describe the disturbance correct to $O(a)$ by means of a time-harmonic acoustic dipole in the usual way (Lighthill 1978a, §1.11) and, further, replace (6.3) by

$$
\nabla \cdot \overline{\mathbf{u}}^{\mathrm{L}}=0
$$

with error $O\left(a^{4}\right)$ since $\overrightarrow{\mathbf{u}}^{\mathrm{L}}=O\left(a^{2}\right)$. Straightforward calculation, using (3.1) in (6.1) to find $\nabla \times \overline{\mathbf{u}}^{\mathrm{L}}$, then shows that the Stokes stream function $\Psi$ for $\overline{\mathbf{u}}^{\mathrm{L}}$ is proportional to

$$
\left(\cos \theta-\cos ^{3} \theta\right)\left\{1-\left(r_{b} / r\right)^{2}\right\}
$$

in spherical co-ordinates with $\theta=0$ on the axis of vibration, and $r=r_{b}$ on the mean boundary. (It is remarkable that the expression (6.5) does not depend, as does the

$\dagger$ An even simpler yet relevant example (Brillouin 1925, 1936) is the one-dimensional problem of acoustic waves generated by an infinite plane boundary vibrating steadily about $x_{1}=0$; clearly $\mathbf{P} \neq 0$ but $\overline{\mathbf{u}}^{\mathrm{L}}=0$ everywhere, by mass conservation $(\S 4)$. 


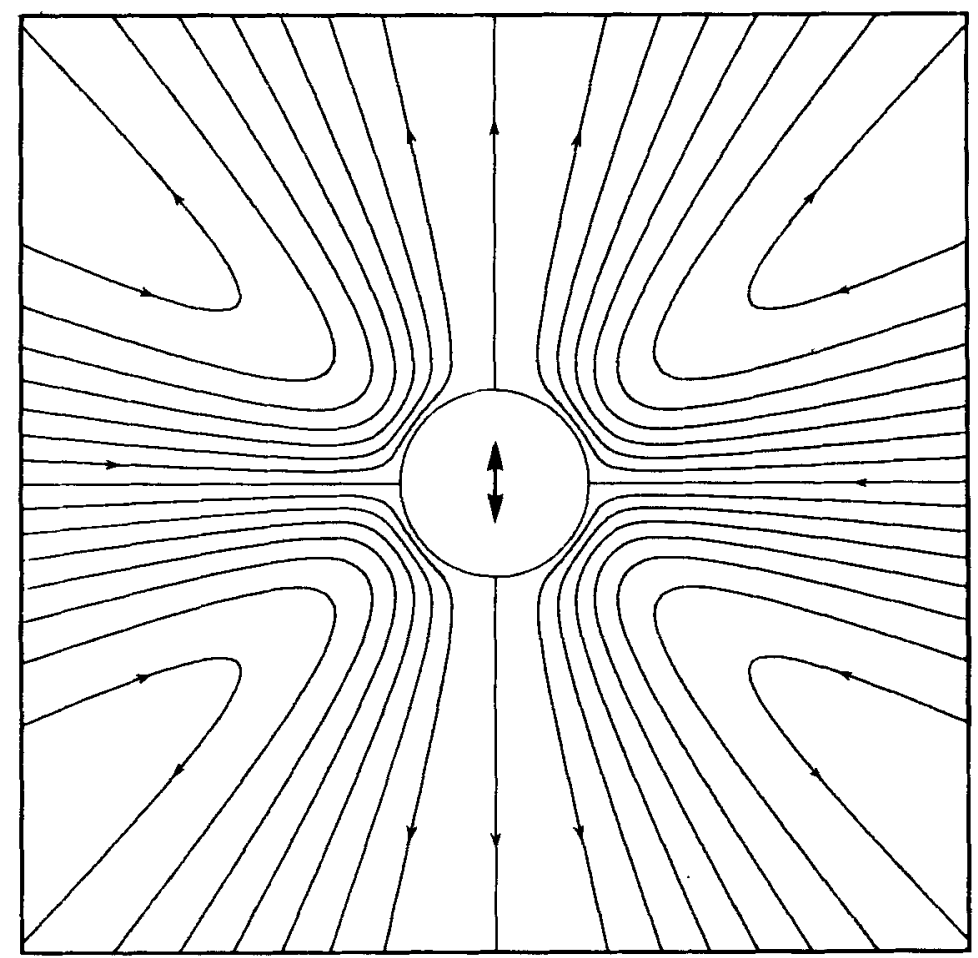

Frgure 3. Steady Lagrangian-mean streaming due to acoustic radiation from a rigid sphere vibrating in the $\theta=0$ direction in an inviscid, homentropic fluid. The expression (6.5) to which the Stokes stream function is proportional is contoured at intervals of 0.05 . The effect of the boundary conditions is significant even in the far field; for instance on the axis $\theta=0$ the far -field value of $\left|\overline{\mathbf{u}}^{\mathrm{L}}\right|$ is only two-thirds of the acoustic energy per unit mass divided by the sound speed.

acoustic field itself, on $\lambda / r_{b}$, where $\lambda$ is the acoustic wavelength.) The coefficient of proportionality for (6.5) is such that the radial component of $\overline{\mathbf{u}}^{\mathrm{L}}$ satisfies

$$
\left(r^{2} \sin \theta\right)^{-1} \partial \Psi / \partial \theta \sim \frac{2}{3} P_{0}
$$

on the axis as $r \rightarrow \infty$, where $\mathrm{p}_{0}(r)$ is the magnitude of $\mathbf{p}$ on the axis in the far field. (There $\mathbf{p}$ is directed radially and $|\mathbf{p}|$ equals the acoustic energy density $\hat{E}$ divided by the sound speed, as is easily shown; see equation (5.10) of the companion paper.)

When $r_{b} / \lambda$ is large, the flow involves a mass flux larger by a factor of order $r_{b} / \lambda$ than that which would arise from viscous boundary-layer streaming (e.g. Schlichting 1932; Stuart 1966; Riley 1967), and in any case extends to larger distances for all values of $r_{b} / \lambda$. In practice, wave dissipation (vitiating $(6.1)$ by making the right-hand side of (3.8) significant in the far field) can produce an even stronger effect extending to yet larger distances, well beyond the wave field itself (Lighthill 1978a, $b$ ).

If, as in the present example, $\overline{\mathbf{u}}$ is $O\left(a^{2}\right),(2.27)$ shows that $\bar{u}_{i}^{\mathrm{S}}$ may be approximated by $\overline{\xi_{j} u_{i, j}^{\prime}}$, which equals $\overline{\xi_{j} u_{j, i}^{\prime}}$ for irrotational flow. Using (2.28) and $(2.10 b)$, we find that $u_{j}^{l} \doteq u_{j}^{\prime}=\xi_{j, t}+O\left(a^{2}\right)$ when $\overline{\mathbf{u}}$ is $O\left(a^{2}\right)$; substitution into (3.1) with $\boldsymbol{\Omega}=\mathbf{0}$ then gives

$$
\mathrm{P}_{i}=\bar{u}_{i}^{\mathrm{S}}-\frac{1}{2}\left(\overline{\xi_{j} \xi_{j}}\right)_{, i t}+O\left(a^{3}\right)
$$

which is consistent with the exact result

$$
\nabla \times\left(\overline{\mathbf{u}}^{\mathrm{S}}-\mathbf{p}\right)=0
$$


for irrotational motion, a corollary of (6.1). Since $(\overline{)}$ denotes a time average here, (6.7) implies that

$$
\mathrm{P}_{i}=\bar{u}_{i}^{\mathrm{S}}+O\left(a^{3}\right) \text {. }
$$

This latter is a familiar approximate result for sinusoidal acoustic and surface-gravity waves. But we emphasize that there is no such relation between $\bar{u}^{s}$ and $p$ in less special circumstances, particularly in the rotational flows usually met with in astrogeophysical fluid dynamics, a simple but sufficient example being the internal-gravity-wave problem referred to in $\$ 5.4$ (McIntyre 1973, p. 810).

\section{The frozen-field transformation, and $\overline{\mathbf{u}}^{\mathrm{L}}-\mathrm{p}$ for irrotational flow}

It can be useful to define further dependent variables in partial analogy to $\tilde{\rho}$ by insisting that they have some simple behaviour, like (4.5), under the mapping $\mathbf{x} \rightarrow \mathbf{\Xi}$. This is the basic idea motivating the work of Soward (1972) on the magnetic induction equation, and in our case will lead to a more direct derivation of the basic result (corollary IV of $\S 3$ ) that $\overline{\mathbf{u}}^{\mathrm{L}}-\mathbf{p}$ is irrotational when $\mathbf{u}(\mathbf{x}, t)$ is irrotational.

For any vector field $\mathbf{V}(\mathbf{x}, t)$ we can define an associated vector $\tilde{\mathbf{V}}$ such that

$$
\int_{\Sigma \xi} V_{j} d \Sigma \Sigma_{j}^{\xi}=\tilde{V}_{j} d \Sigma \Sigma_{j}
$$

where $\Sigma^{\xi}$ is the image of an arbitrary surface $\Sigma$ under the mapping $\mathbf{x} \rightarrow \Xi$. Then $\tilde{\mathbf{V}}$ is that vector field which would be carried into $V^{\xi}$ if the vector field were 'frozen' into an imaginary fluid which suffered the displacement $\mathbf{x} \rightarrow \mathbf{\Xi}$. Equation (7.1) is satisfied (for any mapping $\mathbf{x} \rightarrow \Xi$ ) if we take (Soward, op. cit.; Moffatt 1978, chap. 8)

$$
\tilde{V}_{j}=K_{i j} V_{i}^{\xi}
$$

where $K_{i j}$ is the cofactor of $J$ defined in appendix A. This device is certainly useful when the field $V$ is in fact frozen into the actual fuid motion, in which case its governing equation is

$$
\partial \mathbf{V} / \partial t-\nabla \times(\mathbf{u} \times \mathbf{V})=0 .
$$

Examples are vorticity in an inviscid, homogeneous fluid, and a magnetic field in a perfectly conducting fluid (but not velocity $\mathbf{u}$ or mass flux $\rho \mathbf{u}$ !). When $\mathbf{V}$ satisfies (7.3), $\tilde{\mathbf{V}}$ must satisfy

$$
\partial \tilde{\mathbf{V}} / \partial t-\nabla \times\left(\overline{\mathbf{u}}^{\mathrm{L}} \times \tilde{\mathbf{V}}\right)=0
$$

(Moffatt, op. cit.), just as $\tilde{\rho}$ satisfies (4.2); and under postulate (viii), $\tilde{V}$ is then a mean quantity $(\overline{\tilde{V}}=\tilde{V})$ for essentially the same reason as $\tilde{\rho}$ is [cf. appendix A, $\S$ A 2]. However, with dissipation or external forcing, the right-hand side of (7.3), unlike that of (4.2), becomes different from zero; and $\tilde{V}$ is not generally a mean quantity. The use of $\tilde{\mathbf{V}}$ may nevertheless lead to powerful results, as Soward's work shows.

When $\mathbf{V}=2 \boldsymbol{\Omega}+\nabla \times \mathbf{u}$, the absolute vorticity, (7.2) implies that

$$
\overline{\tilde{\mathbf{V}}}=2 \boldsymbol{\Omega}+\nabla \times\left(\overline{\mathbf{u}}^{\mathrm{L}}-\mathbf{p}\right) .
$$

[We are indebted to Dr Soward for pointing out this result, which corresponds directly to the transformation of $\nabla_{\mathbf{x}} \times \mathbf{u}^{*}$ in his equation (5.1) into $\nabla \times \mathbf{G}$ in his equation (5.4).] The derivation is indicated in (A 13)-(A 17). It follows that if $\mathbf{u}$ is irrotational and 
$\boldsymbol{\Omega}=0$, then $\overline{\hat{\mathbf{V}}}=0$ and $\overline{\mathbf{u}}^{\mathrm{L}}-\mathbf{p}$ is irrotational; this constitutes a more direct proof of corollary IV of $\S 3$.

\section{On the radiation-stress concept}

One of the basic relationships generally obscured by the Eulerian-mean description is that between the effective momentum transfer due to waves, and the wave drag on a boundary generating the waves (Bretherton 1969a,b, 1971; Grimshaw 1975; Müller 1976; McIntyre 1977, 1978). The present theory provides a natural framework for expressing this idea and assessing its domain of validity in any given case. The basis of the necessary analysis is as follows; see also $\S 5.2$ of the companion paper.

\subsection{A form of the mean-flow equations analogous to the Eulerian-mean equations}

The usual Eulerian-mean equations are the result of simply applying the operator $(\bar{r} \text { to the equations for the total motion. If instead we apply the operator } \overline{(})^{\mathrm{L}}$ to the general equation of motion (3.2), with $j$ replaced by $i$, the pressure term becomes

$$
\left.\overline{\left(\rho^{-1} p_{, i}\right.}\right)^{\mathrm{L}}=\overline{\left(\rho^{-1} p_{, i}\right)^{\bar{\xi}}}=\tilde{\rho}^{-1} \overline{\left(p^{\overline{5}}\right)_{, j} K_{i j}}
$$

by (4.3), (4.6) and (A 4), where $K_{i j}$ is the $(i, j)$ th cofactor of the Jacobian $J$. We write (8.1) as

$$
\begin{array}{cc} 
& \tilde{\rho}^{-1} \bar{P}_{i j, j}, \\
\text { where } & P_{i j}=p^{\xi} K_{i j}
\end{array}
$$

and where (A 3) has been used. By substituting (A $1 b)$ into (8.3) we get

$$
P_{i j}=p^{\xi} J \delta_{i j}-p^{\xi} \xi_{m, i} K_{m j}
$$

The average of this may be rearranged slightly as

where

$$
\bar{P}_{i j}=\bar{p}^{\mathrm{L}} \delta_{i j}-R_{i j}
$$

$$
R_{i j}=\overline{p^{\xi}(1-J)} \delta_{i j}+\overline{p^{\xi} \xi_{m, i} K_{m j}}
$$

Thus the generalized Lagrangian mean of (3.2) may be written

$$
\bar{D}^{\mathrm{L}} \bar{u}_{i}^{\mathrm{L}}+2\left(\Omega \times \overline{\mathbf{u}}^{\mathrm{L}}\right)_{i}+\bar{\Phi}_{, i}+\tilde{\rho}^{-1}\left(\bar{p}^{\mathrm{L}}\right)_{, i}+\bar{X}_{i}^{\mathrm{L}}=-\left(\overline{\Phi_{, i}}\right)^{\mathrm{S}}+\tilde{\rho}^{-1} R_{i j, j} .
$$

Equations (2.21), (5.3) and (4.2) are respectively

and

$$
\begin{gathered}
\bar{D}^{\mathrm{L}} \bar{S}^{\mathrm{L}}+\bar{Q}^{\mathrm{L}}=0, \\
\tilde{\rho}-F\left(\bar{S}^{\mathrm{L}}, \bar{p}^{\mathrm{L}}\right)=\Delta \\
\bar{D}^{\mathrm{L}} \tilde{\rho}+\tilde{\rho} \nabla \cdot \overline{\mathbf{u}}^{\mathrm{L}}=0,
\end{gathered}
$$

where $\Delta$ is given by (5.4). The terms

$$
\left.\tilde{\rho}^{-1} R_{i j, j}, \quad \overline{(\nabla \Phi)}\right)^{\mathrm{S}}, \Delta
$$

on the right of these equations are all wave properties. It may sometimes be appropriate to include also on the right-hand sides wave properties which contribute to $\overline{\mathbf{X}}^{\mathrm{L}}$ and $\bar{Q}^{\mathrm{I}}$, such as $\overline{\mathbf{X}}^{\mathrm{S}}$ and $\bar{Q}^{\mathrm{S}}$, depending on how $\mathbf{X}$ and $Q$ are to be specified in a given application. 


\subsection{The physical meaning of $R_{i j}$}

The tensor $R_{i j}$ is the counterpart of the Reynolds stress or wave-induced excess momentum flux which appears in Eulerian-mean formulations. As is now well known, there is no reason why $R_{i j}$ should equal the Reynolds stress; and this difference, taken together with the absence of a wave-induced forcing term in the mean entropy equation $(8.7 b)$ when $Q=0$, and the presence of the new wave-induced forcing terms $\Delta$ and $(\overline{\nabla \Phi})^{\mathrm{S}}$ (the latter having no counterpart at all in an Eulerian-mean formulation), completes our remarks on the differences between the Eulerian-mean and generalized Lagrangian-mean descriptions begun in $\$ 2.4$.

To see in physical terms how the anisotropic mean stresses $P_{i j}$ and $R_{i j}$ arise, and why $R_{i j}$ differs from the Reynolds stress, note from (A 15) that

$$
\int_{\Sigma} P_{i j} d \Sigma_{j}=\int_{\Sigma \xi} p d \Sigma_{i}^{\xi}
$$

where $\Sigma$ and $\Sigma^{\xi}$ are any pair of surfaces related by the mapping $\mathbf{x} \rightarrow \mathbf{x}+\boldsymbol{\xi}$. (For instance if $\Sigma$ is a plane surface like $\mathscr{S}$ in figure 2 , then $\Sigma^{\mathscr{E}}$ might look like the corrugated boundary there.) Contributions to $\bar{P}_{i j}$ and therefore to $-R_{i j}$ representing forces tangential to $\mathscr{S}$ can arise because pressure fluctuations may be correlated with different slopes of the surface $\Sigma^{\xi}$ (Bretherton 1971). In the example of figure 2, $R_{13}>0$ at the boundary. In the same example, the Reynolds-stress component $-\bar{\rho} \overline{u^{\prime} w^{\prime}}>0$ also; but it is not even approximately equal to $R_{13}$, if only because of the mean Coriolis force on the fluid lying between $\mathscr{S}$ and the boundary (Bretherton 1969a).

\subsection{The asymmetry of $R_{i j}$}

The tensor $\xi_{i, j}$ is not generally symmetric, so $\bar{P}_{i j}$ and $R_{i j}$ are likewise asymmetric despite their role as stress tensors for the mean flow. No paradox is involved, for reasons first indicated by Jones (1973); the usual argument concerning the sum of the moments of surface forces on a fluid element is vitiated by extra contributions which arise as follows. First, the line of action of any resultant body force such as gravity, on a volume $\mathscr{V}^{\xi}$ of fluid, generally suffers an $O\left(a^{2}\right)$ mean displacement because of the disturbance; the associated torque can, alternatively and more easily, be thought of as due to the disturbance 'buoyancy forces' at the edges of $\mathscr{V}^{5}$, as suggested in figure 4. This and a further torque attributable to Coriolis forces are comparable in magnitude with the surface torque on $\mathscr{V}^{\xi}$ represented by the antisymmetric part of $R_{i j}$. Second, there is an effect (which in Jones' case is smaller in order of magnitude) due to the wave-associated contribution to the rate of change of angular momentum of the fluid within $\mathscr{V}^{\xi}$ about a fixed point (see also Bretherton 1978).

\section{4. $R_{i j}$ and the radiation-stress concept}

$R_{i j}$ is closely related to the 'radiation-stress' tensors defined by Brillouin (1925), Longuet-Higgins \& Stewart (1964), Dewar (1970), Bretherton (1971) and others. The relation usually turns out to be much closer than that with the Reynolds stress, and is thus useful in a wider class of examples. In order to be called a radiation stress, as the term has come to be used, $R_{i j}$ must represent the sole effect of the waves on the mean flow. Our theory shows that this generally involves approximation; and the question of whether or not the other effects $\Delta$ and $\overline{(\nabla \Phi)}$ s can be neglected, perhaps 


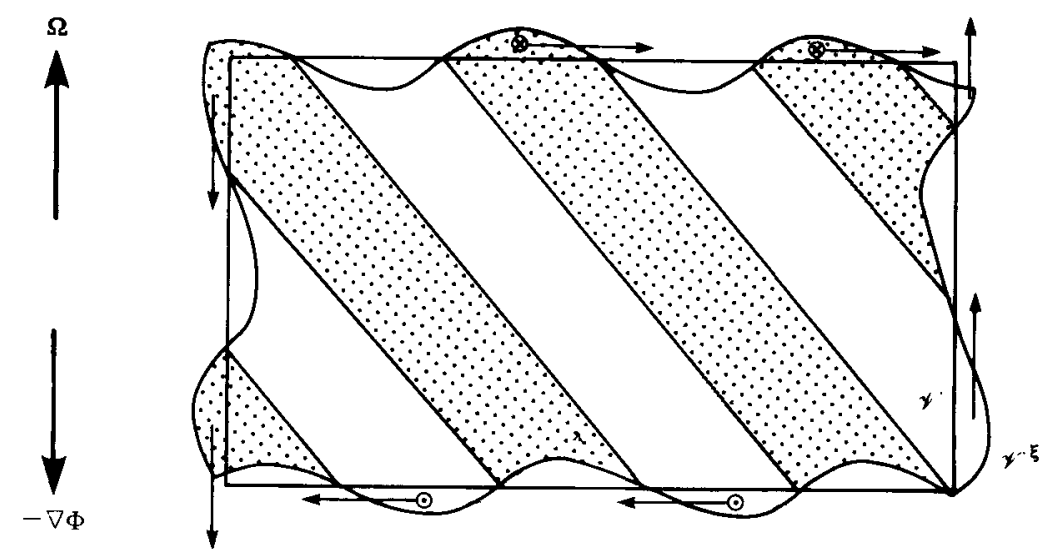

Figure 4. Buoyancy and Coriolis torques on a volume $\mathscr{F}$ distorted by the plane, progressive inertio-gravity waves whose generation is suggested in figure 2. (These torques account for the asymmetry of $R_{i j}$ in this case.) The sloping lines are lines of constant phase. At the instant depicted, the shaded fluid is moving into the paper, and the buoyancy force on it is downward. Since this temporarily 'heavy' fluid is all displaced to the left (and the 'light' fluid in between to the right), the buoyancy forces exert a net anticlockwise torque on $\mathscr{V}^{\prime} \xi$. There is also a clockwise torque on $\mathcal{V}^{\bar{\xi}}$, owing to the Coriolis forces at its top and bottom edges. Attention was first drawn to effects of this kind by Jones (1973).

after integration across a waveguide, is a matter for detailed investigation in each case. Obviously $\left(\overline{\nabla \Phi)^{\mathrm{s}}}\right.$ is zero in models postulating a time-independent, spatially uniform gravitational-centrifugal acceleration $\nabla \Phi$; but there is seldom any reason why $\Delta$ should be exactly zero.

Both $\Delta$ and $(\overline{\nabla \Phi})^{\mathrm{s}}$ are usually negligible, however, for almost-plane waves in an incompressible fluid $(F(S, p)=F(S))$; in such cases $R_{i j}$ can immediately be given the status of a radiation stress, in the first approximation. A well-known example in which this is true but a corresponding statement not true of the Reynolds stress is that of almost-plane inertio-gravity waves (Grimshaw 1975; Müller 1976; McIntyre 1977, 1978). In that example, the extra effect on the Eulerian-mean flow represented by the buoyancy-foreing term on the right of (2.29) is significant in the first approximation, but the terms $\Delta$ and $(\overline{\nabla \Phi})^{\mathrm{s}}$ are negligible in $(8.7)$.

In acoustics $\Delta$ is not at all negligible, even for plane waves. However, the radiation. stress idea is rescued (e.g. Brillouin 1925; Bretherton 1971) by redefining 'mean pressure' in such a way that $\Delta$ disappears from $(8.7 c)$. A compensating $O\left(a^{2}\right)$ change is then required in the definition of the isotropic part of $R_{i j}$, which then becomes the familiar acoustic radiation stress and does represent the sole effect of the waves on the newly-defined mean flow. $\dagger$ This device for eliminating $\Delta$ is possible in principle

$\dagger$ Correct to $O\left(a^{2}\right)$, the result of this procedure is that the isotropic term $\overline{p^{\xi}(1-J)} \delta_{i j}$ in the expression (8.6) for $R_{i j}$ is replaced by a term $-c^{-3}(\partial c / \partial \rho)_{S} \overline{p^{\prime 2}} \delta_{i j}$ (Brillouin 1925; Bretherton 1971), where the sound speed $c=\{\partial F(S, p) / \partial p\}^{-\frac{1}{2}}$ is evaluated for the basic state correct to zeroth order in $a$, and where plane periodic waves are assumed so that $\bar{T}_{j j}=0$ in (A 9), by (A 11), and

$$
\xi_{k, k} \doteq-\rho^{l} / \rho \doteqdot-p^{l} / \rho c^{2} \doteq-p^{\prime} / \rho c^{2} .
$$

The term $-c^{-3}(\partial c / \partial \rho)_{S}{\overline{p^{\prime 2}}}_{i j}$, which in terms of the usual acoustic energy density $\hat{E}$ becomes $-(\rho E / c)(\partial c / \partial \rho) \delta_{i j}$, comes entirely from elimination of the $F$ terms in (5.4), i.e. from nonlinearity of the equation of state (the 'hard-spring effect'). Elimination of the other contribution to $\Delta$, $\tilde{\rho}-\bar{\rho}^{\mathrm{L}}$, accounts for the disappearance of $\overline{p^{\bar{\xi}(1-J)}} \delta_{i j}$. 
whenever the fluid is compressible, and is formally analogous to the usual procedure for dealing with 'bulk viscosity' in the Navier-Stokes equations (e.g. Batchelor 1967, p. 154). In Longuet-Higgins \& Stewart's case of surface-gravity waves $\Delta$ is again significant but can be eliminated, the relevant 'compressibility' being that of the two-dimensional, vertically-integrated, free-surface flow.

A very simple example where $\Delta$ can neither be neglected to leading order nor eliminated in any useful sense is that of guided internal gravity waves ( $\$ 5.4 ;$ McIntyre 1973 , p. 810). For incompressible or nearly incompressible flow, an attempt to eliminate $\Delta$ by the foregoing device would of course lead to an infinite or unphysically large 'mean pressure'. Another such example is the Boussinesq model of equatorial planetary waves to be discussed in $\S 9$.

\subsection{Relation to earlier theories}

Many alternative ways of writing $R_{i j}$ can be constructed from the two identities (A $1 b$ ) and (A 8), and the identity

$$
K_{i j}=\left(1+\xi_{m, m}\right) \delta_{i j}-\xi_{j, i}+k_{i j},
$$

where $k_{i j}$ is the $(i, j)$ th cofactor of $\xi_{i, j}$. We quote one exact expression which is convenient in practice, and which shows how the present theory is related to earlier, approximate theories. It may be obtained by manipulating (8.6) with the aid of (8.10) and (A 8):

where

$$
R_{i j}=-\overline{\left(\xi_{k, k} p^{l}+\frac{1}{2} T_{k k} p^{\overline{5}}\right)} \delta_{i j}+\overline{\left(p^{l}-\xi_{m}\left(\bar{p}^{\mathrm{L}}\right)_{, m}\right\} \xi_{j, i}}+Z_{i j}+N_{i j}+\overline{p^{l} T_{i j}}
$$

$$
\begin{aligned}
Z_{i j} & =-\bar{p}^{\mathrm{L}}\left(\overline{\xi_{m} \xi_{j, m}}\right)_{, i}+\frac{1}{2}\left\{\bar{p}^{\mathrm{L}}\left(\overline{\xi_{m} \xi_{j}}\right)_{, i}\right\}_{, m}, \\
N_{i j} & =\frac{1}{2}\left\{\bar{p}^{\mathrm{L}}\left(\overline{\left.\xi_{m} \xi_{j, i}-\xi_{j} \xi_{m, i}\right)}\right\}_{, m},\right.
\end{aligned}
$$

and $T_{i j}$ is defined by (A 10). $T_{i j}$ is $O\left(a^{2}\right)$ so $\overline{p^{l} T_{i j}}$ is $O\left(a^{3}\right)$ for small $a$; and $N_{i j}$ can be ignored being identically non-divergent $\left(N_{i j, j}=0\right)$. To $O\left(a^{2}\right)$, the $\delta_{i j}$ term in (8.11) equals that in (8.6).

If $\left(\bar{l}\right.$ is a space average in the (Cartesian) $i$ direction, $Z_{i j}$ vanishes. Thus $Z_{i j}$ is often negligible in problems of almost-plane waves, an example being that considered by Grimshaw (1975). In such cases the wave-induced flux $-R_{i j}$ of $i$-momentum, in some direction $j$ orthogonal to $i$, is given by minus the second term in (8.11) alone. To leading order, that term becomes

$$
\overline{p^{\prime} \xi_{j, i}}+O\left(a^{3}\right)
$$

since by (2.28), $p^{l}-\xi_{m}\left(\bar{p}^{\mathrm{L}}\right)_{, m}=p^{\prime}+O\left(a^{2}\right)$, to leading order the Eulerian pressure disturbance. This is the form arising in Grimshaw (op. cit.), in I (equations A 11), and in (9.1) below. The fact that $p^{\prime}$ is involved, rather than $p^{l}$, is noteworthy in view of the above-mentioned fact that $R_{i j}$ itself represents the wave-induced part of the mean pressure force across a disturbed material surface. On such a surface $p^{l}$ would appear at first sight a more natural measure of the disturbance pressure. However (8.11) and (8.12) show clearly that $p$ ' is the part of the pressure disturbance that 'matters' to leading order, when $i \neq j$, under the kinds of approximation often used in wave theories. In the important case of Boussinesq, stratified flow $p^{\prime}$ is of course a more convenient entity to deal with than $p^{l}$. The latter is dominated by the effect of vertical 
displacement through the large, hydrostatic pressure gradient, which is not explicitly represented in the equations once the Boussinesq approximation has been introduced.

\section{Approximate equations for incompressible, Boussinesq flow, and the model of equatorial planetary waves on a beta-plane studied in I}

Because of the inconvenience of using the total pressure when the Boussinesq approximation is appropriate, it can be useful in practice to apply our procedures directly to the Boussinesq equation of motion. That equation has the same form as (3.2), except that the term $\Phi_{, j}$ is replaced by an upward-directed 'buoyancy accelera$\operatorname{tion}^{\prime} \theta_{j}(\mathbf{x}, t)$, and $\rho$ is taken constant in the term $\rho^{-1} p_{, j}$. The symbol $p$ now denotes the excess pressure relative to a basic hydrostatic pressure distribution associated with the constant density $\rho$; that is, $p$ now denotes what would previously have been written as $p+\rho \Phi$ (with $\rho$ exactly constant).

Thus the results of $\S 3$, for instance, apply immediately in the Boussinesq limit, provided we interpret $p$ as the modified pressure just defined, replace $\nabla \Phi$ by the buoyancy acceleration $\theta$, and note that $\theta$ is proportional to excess entropy times $\nabla \Phi$. The departure $q$ from adiabatic motion defined by (3.5) becomes simply proportional to $-S^{l}$ (ef. I, equation $5.3 b$ ), because the first term in (3.5) may be replaced by its first-order Taylor expansion about $\bar{S}^{\mathrm{L}}$, even for finite-amplitude disturbances, in the Boussinesq limit. A useful consequence is that $\bar{q}=0$, so that $p^{\xi}$ may immediately be replaced by $p^{l}$ on the right of (3.8) and (3.11).

It is also of interest to apply the operator ()$^{\mathrm{L}}$ directly to the Boussinesq equation of motion and compare the result with that obtained in $\$ 8$. Using (2.15) and replacing $j$ by $i$ in (3.2) we get the following Lagrangian-mean equation of motion:

$$
\bar{D}^{\mathrm{L}} \bar{u}_{i}^{\mathrm{L}}+\mathbf{2}\left(\boldsymbol{\Omega} \times \overline{\mathbf{u}}^{\mathrm{L}}\right)_{i}-\bar{\theta}_{i}^{\mathrm{L}}+\rho^{-1}\left(\bar{p}^{\mathrm{L}}\right)_{, i}+\bar{X}_{i}^{\mathrm{L}}=\rho^{-1}\left(\overline{p^{\prime} \xi_{j, i}}\right)_{, j}+\frac{1}{2} \rho^{-1}\left(\overline{\xi_{j} \xi_{k}}\right)_{, i} \bar{p}_{, j k}+O\left(a^{3}\right),
$$

after a little manipulation using (2.27) on the pressure-gradient term, and the fact that when $\nabla \cdot \mathbf{u}^{\prime}=0$,

$$
\nabla . \xi=O\left(a^{2}\right)
$$

as we shall show shortly. The first term on the right of (9.1) corresponds to (8.12). The second term, however, is not a divergence; to obtain an equation in which the wave-forcing term is the divergence of a tensor as in $\tilde{\rho}$ times $(8.7 a)$, multiply both sides of (9.1) by

$$
J=\tilde{\rho} / \rho=1-\frac{1}{2}\left(\overline{\xi_{j} \xi_{k}}\right)_{j k}+O\left(a^{3}\right),
$$

take an $O\left(a^{2}\right)$ term $\left.-\frac{1}{2} \rho^{-1} \bar{p}_{, i} \overline{(\xi}_{j} \xi_{k}\right)_{j k}$ over to the right, using the alternative (original) form $\rho^{-1}(\overline{\nabla p})^{\mathrm{L}}$ for the pressure terms in $(9.1)$ itself.

The relations (9.2) and (9.3) follow from the fact that $\rho$ and $\rho^{\xi}$ tend to the same constant value in the Boussinesq, incompressible limit, so that (4.3) and (4.6) imply that $\tilde{\rho} / \rho \rightarrow J \rightarrow \bar{J}$. Then (9.2) follows by comparing (A 8) and (A 9); and since the first term in (A 11) becomes negligible we also get (9.3), from (A 9) and the fact that $\tilde{\rho} / \rho=\bar{J}$.

It is noteworthy that, in virtue of (9.3) and (4.2),

$$
\nabla \cdot \overline{\mathbf{u}}^{\mathrm{L}}=\frac{1}{2} \bar{D}^{\mathrm{L}}\left(\overline{\xi_{j} \xi_{k}}\right)_{j k}+O\left(a^{3}\right) \neq 0 .
$$

In an incompressible fluid the disturbance may force the Lagrangian-mean flow to be divergent. This does not contradict the fact that the Jacobian for the conventional Lagrangian description of the total motion is unity for incompressible flow, since that 
Jacobian is the product of $J$ with the Jacobian for the Lagrangian description of the mean motion [Eckart 1963, equation (2.5)]; the two factors need not separately be unity. $\uparrow$ The generally divergent character of the Lagrangian-mean flow is rather obvious in some cases; for instance if the centre of mass of the tube of fluid depicted in figure $1(b)$ is initially very close to a stationary boundary parallel to $x_{1}$, it will tend to move away from the boundary as the disturbance grows.

Like the second term of the Stokes correction (2.27), the right-hand side of (9.4) can often be neglected in problems of 'almost-plane' waves [Bretherton 1971, p. 88, note (i)]. But it is again crucial in transient problems involving waveguide structure; an example is classical surface gravity waves. Other examples include the guided internal gravity wave problem mentioned in $\$ 5.4$, and the equatorial planetary wave problem studied in I. In view of its current scientific interest (see I for background), we sketch a few details for the latter problem.

In the simplest Boussinesq, 'beta-plane' formulation of the problem, $\overline{(})$ is the average with respect to a Cartesian co-ordinate $x_{1} ; x_{3}$ is directed 'upward' and $x_{2}$ 'northward', $\boldsymbol{\theta}=(0,0, \theta)$, and $2 \boldsymbol{\Omega}=\left(0,0, \beta x_{2}\right)$ with $\beta$ constant; note that

$$
\overline{2(\boldsymbol{\Omega} \times \mathbf{u})^{\mathrm{L}}} \neq 2 \Omega \times \overline{\mathbf{u}}^{\mathrm{L}} .
$$

In fact a little manipulation using (2.27) shows that

$$
2 \overline{(\boldsymbol{\Omega} \times \mathbf{u})^{\mathrm{L}}}=2 \boldsymbol{\Omega} \times \overline{\mathbf{u}}^{\mathrm{L}}+\left\{-\frac{1}{2} \beta\left({\overline{\xi_{2}^{2}}}_{, t}, \quad \beta \overline{\xi_{2} u_{1}^{l}}, \quad 0\right\}+O\left(a^{3}\right)\right.
$$

in this case. On a beta-plane this expression must be used in place of the Coriolis term both in (9.1) and in theorem $I$ and its corollaries. The $O\left(a^{2}\right)$ Lagrangian-mean meridional circulation $\left(\bar{u}_{2}^{\mathrm{L}}, \bar{u}_{3}^{\mathrm{L}}\right)$ is governed by (9.4) with $\bar{D}^{\mathrm{L}} \doteq \partial / \partial t$, together with $(5.5 d)$ with buoyancy $\theta$ in place of entropy $S$, viz.

$$
\bar{\theta}_{, t}^{\mathrm{L}}+\bar{\theta}_{, 2}^{\mathrm{L}} \bar{u}_{2}^{\mathrm{L}}+\bar{\theta}_{, 3}^{\mathrm{L}} \bar{u}_{3}^{\mathrm{L}}=-\bar{Q}^{\mathrm{L}} .
$$

Under the approximations described in detail in $\S 6$ of I it turns out that $\partial \bar{\theta}^{\mathrm{s}} / \partial t \gg \partial \bar{\theta} / \partial t$, so the mean buoyancy equation becomes

$$
\bar{\theta}_{, 2}^{\mathrm{L}} \bar{u}_{2}^{\mathrm{L}}+\bar{\theta}_{, 3}^{\mathrm{L}} \bar{u}_{3}^{\mathrm{L}} \doteq-\partial \bar{\theta}^{\mathrm{S}} / \partial t-\bar{Q}^{\mathrm{L}}
$$

It also turns out that in calculating both $\bar{\theta}^{\mathrm{s}}$ and $\bar{Q}^{\mathrm{s}}$ the right-hand side of (2.27) may be approximated by $\overline{\left(\bar{\xi}_{2} \varphi^{\prime}\right)_{2}}$ and that the term $\bar{\theta}_{, 2}^{\mathrm{L}} \bar{u}_{2}^{\mathrm{I}} \mathrm{L}$ may be neglected in (9.6), as may $\frac{1}{2}\left(\overline{\xi_{3}^{2}}\right)_{33 t}$ in (9.4). Finally $\bar{\theta}_{, 3}\left(\doteq \bar{\theta}_{, 3}^{\mathrm{L}}\right)$, representing the basic stable stratification, can be treated approximately as a function of $x_{3}$ alone, and $\theta^{\prime} \doteq-\bar{\theta}_{3} \xi_{3}$. Hence if the Eulerian mean of $Q$ is zero (as was, somewhat arbitrarily, assumed in I), the equations are satisfied by

and

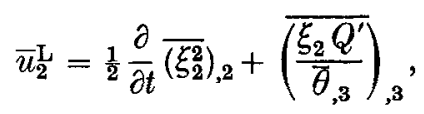

$$
\bar{u}_{3}^{\mathrm{L}}=\frac{\partial}{\partial t} \overline{\left(\xi_{2} \xi_{3}\right)_{, 2}}-\overline{\left(\frac{\xi_{2} Q^{\prime}}{\bar{\theta}_{, 3}}\right)_{, 2}},
$$

to the level of approximation asserted in $\S 6 c$ of $\mathrm{I}$. The divergence

$$
\bar{u}_{2,2}^{\mathrm{L}}+\bar{u}_{3,3}^{\mathrm{L}} \doteqdot \partial\left\{\frac{1}{2}\left(\overline{\xi_{2}^{2}}\right)_{, 22}+\left(\overline{\xi_{2} \xi_{3}}\right)_{, 23}\right\} / \partial t
$$

is significantly different from zero unless the waves are steady. We have checked that these results, together with the appropriate approximations in corollary I [equation

+ It is now clear why, even for incompressible flow, our mapping $\mathbf{x} \rightarrow \mathbf{x}+\boldsymbol{\xi}$ satisfying (2.7) and (2.8) does not belong to the class considered by Soward (1972), who demanded that $J=1$. 
(3.9), with the Coriolis term replaced by (9.5)], and use of both terms in (2.27) for $\bar{u}_{1}^{\mathrm{s}}$, do lead to the result (7.1) of I for $\partial \bar{u}_{1} / \partial t$. This is an excellent check on the correctness of that result, since in $I$ it was derived via a very different route.

It may be shown that the Coriolis force $\left(2 \Omega \bar{u}_{2}^{\mathrm{L}}\right)$ associated with (9.7) makes a contribution to $\bar{u}_{1, t}^{\mathrm{L}}$ of the same order of magnitude as that due to $R_{1 j, j}$, a point relevant to the discussion of limitations on the radiation-stress concept in $\S 8.4$. It is also of interest that $\bar{\theta}^{\mathbf{L}}$ and $\bar{u}_{1}^{\mathrm{L}}$ are significantly out of 'thermal-wind balance', mainly because of the contribution $\overline{\left(\xi_{2,3} p^{\prime}\right)_{, 2}}$ to the vertical force on the right of the third component of (9.1).

\section{Concluding remarks}

Although no formal assumption limits either the amplitude or the nature of the disturbance in our theory the complexity of real flows will, of course, dictate practical limitations. For instance dispersive waves of dimensionless amplitude $a \gtrsim 1$ are as a rule strongly unstable to many other scales of motion, leading to sporadic or fullydeveloped turbulence. It would be impractical to compute the $\xi$ and $\overline{\mathbf{u}}^{\mathrm{L}}$ fields associated with such a motion in all its detail. Cases must be anticipated where $\max |\xi|$ would tend to increase indefinitely with time. Turbulent motion is not necessary for this; it will happen, for instance, with laminar 'rotor' or 'cats-eye' patterns such as are obtained in idealized models of large-amplitude waves, or of small-amplitude waves at critical levels. The point can be appreciated by visualizing the fate of the line of particles in figure 1. (Unboundedness of $\xi$ need not, of course, imply unboundedness of $\overline{\mathbf{u}}^{\mathrm{L}}$, as is clear from (2.1) and the example of a two-dimensional, ultimately steady cats-eye pattern, for which each initially-streamwise line of particles will evidently remain within some region throughout which $\mathbf{u}^{\xi}$ is bounded.)

Against such possible restrictions on applicability must be set the very great simplification and unification of theoretical structure offered by the generalized Lagrangian-mean description. Exact results such as (6.1)-(6.3) extend and put into perspective various classical results for irrotational waves; and general theorems of the type given in $\$ 3$ have already found significant applications to large-scale geophysical phenomena. In I we showed how, as well as giving qualitative insight, such general results can drastically simplify the explicit calculation of mean-flow evolution in a particular case (namely equatorial planetary waves). This is reminiscent of the simplification in Braginskii's dynamo calculations resulting from the use of Soward's formalism (Moffatt 1978). The results of I revealed an interesting dependence of mean-acceleration profiles upon the wave dissipation mechanism, which had not been appreciated before; the GLM description evidently provides a far more direct route to such results than does the conventional Eulerian-mean description, especially when the generalization to finite amplitude is of interest (cf. the far more complicated manipulations involved in the Eulerian analysis of III, even at $O\left(a^{2}\right)$ ). In the companion paper we shall find moreover that the GLM description leads to a remarkable simplification in the exact theory of 'wave-action' and related conservable wave properties. $\dagger$

$\dagger$ Note added in proof: Another example in which use of the GLM theory drastically simplifies a mean-flow calculation has recently been given by Grimshaw (1978). 
We thank F. P. Bretherton for comparing notes on generalized Lagrangian-mean formulations, and A. M. Soward, whose penetrating review of the first version of this paper helped us to appreciate the partial relation with his earlier work on dynamos and greatly influenced the final presentation, most particularly that of $\S \S 3$ and 7 . T. Matsuno, P. B. Rhines, E. A. Spiegel and M. E. Stern provided further helpful comments and inspiration. D.G.A. thanks Trinity College, Cambridge, the Natural Environment Research Council, and the National Science Foundation (grant NSF-g76-20070 ATM) for financial support.

\section{Appendix A. Manipulations involving the Jacobian $J$ and its cofactors $K_{i j}$}

A 1. Direct proof that $\tilde{\rho}$ satisfies (4.2)

We now prove by direct manipulation that $\tilde{\rho}$ satisfies (4.2), and at the same time introduce a few identities which will be needed elsewhere. Let $K_{i j}$ be the cofactors of $J$, which satisfy

$$
\Xi_{i, k} K_{i j}=J \delta_{k j}=\Xi_{k, i} K_{j i}
$$

Since $\Xi_{i, k}=\delta_{i k}+\xi_{i, k}$, the first of these is equivalent to

We also have

$$
K_{k j}=J \delta_{k j}-\xi_{i, k} K_{i j}
$$

$$
K_{i j}=\partial J / \partial \Xi_{i, j}=\frac{1}{2} \epsilon_{i l m} \epsilon_{j p q} \Xi_{l, p} \Xi_{m, q},
$$

whence it should be noted for later reference that

Next, the inverse of $(2.4 b)$ is

$$
K_{i j, j}=0 .
$$

Now (A $2 a$ ) implies that

$$
\begin{aligned}
\left(\varphi_{, i}\right)^{\xi} & =\left(\varphi^{\xi}\right)_{, j} K_{i j} / J . \\
J_{, \mu} & =K_{i j}\left(\Xi_{i, j}\right)_{, \mu},
\end{aligned}
$$

where ( ),$\mu$ stands for $\partial / \partial t$ or $\partial / \partial x_{k}$. Hence

$$
\bar{D}^{\mathrm{L}} J=K_{i j} \bar{D}^{\mathrm{L}} \Xi_{i, j}=K_{i j}\left(\left(\bar{D}^{\mathrm{L}} \Xi_{i}\right)_{, j}-\bar{u}_{k, j}^{\mathrm{L}} \Xi_{i, k}\right\}=\left(u_{i}^{\mathrm{L}}\right)_{, j} K_{i j}-\bar{u}_{k, j}^{\mathrm{L}} \delta_{j k} J,
$$

using (2.10a) and (A 1a). By (A 4) the term in $K_{i j}$ equals $J\left(u_{i, i}\right)^{\xi}=J(\nabla . \mathbf{u})^{\xi}$; hence

By (2.14),

$$
\bar{D}^{\mathrm{L}} \boldsymbol{J}=J\left\{(\nabla \cdot \mathbf{u})^{\xi}-\nabla \cdot \overline{\mathbf{u}}^{\mathrm{L}}\right\} .
$$

Thus

$$
\bar{D}^{\mathrm{L}}\left(\rho^{\check{\zeta}}\right)=(D \rho / D t)^{\xi} \text {. }
$$

$$
\bar{D}^{\mathrm{L}}(\tilde{\rho})=\bar{D}^{\mathrm{L}}\left(\rho^{\xi} J\right)=(D \rho / D t)^{\xi} J+\rho^{\xi} J\left\{(\nabla \cdot \mathbf{u})^{\xi}-\nabla \cdot \overline{\mathbf{u}}^{\mathrm{L}}\right\},
$$

whence (4.2) follows upon using (4.1).

\section{A 2. Direct proof that $\tilde{\rho}$ is a mean quantity}

By applying $(\zeta$ to (4.2) and using (i)-(iv), we see that (4.2) is true with $\overline{\tilde{\rho}}$ in place of $\tilde{\rho}$. Therefore

so that

$$
\begin{gathered}
\overline{\tilde{\rho}}^{-1} \bar{D}^{\mathrm{L}} \overline{\tilde{\rho}}=-\nabla \cdot \overline{\mathbf{u}}^{\mathrm{L}}=\tilde{\rho}^{-1} \bar{D}^{\mathrm{L}} \tilde{\rho}, \\
\bar{D}^{\mathrm{L}}(\ln \overline{\tilde{\rho}}-\ln \tilde{\rho})=0 .
\end{gathered}
$$


Equation (4.6) then follows by integration along mean trajectories since postulates (vi) and (viii) imply that at some point $\left(\mathbf{x}_{0}, t_{0}\right)$ on each mean trajectory $\rho^{\prime}=0, \xi=0$, $J=1, \rho=\bar{\rho}=\rho^{\xi}=\rho^{\xi} J=\overline{\rho^{\xi}} \bar{J}$, i.e. $\tilde{\rho}=\overline{\tilde{\rho}}$.

\section{A 3. Further properties of $J$ and $K_{i j}$}

The Jacobian may be expanded as

$$
J=\frac{1}{6} \epsilon_{i l m} \epsilon_{j p q} \Xi_{i, j} \Xi_{l, p} \Xi_{m, q}=1+\xi_{j, j}+\frac{1}{2} T_{j j}+\operatorname{det}\left(\xi_{i, j}\right),
$$

so by (2.7) and postulate (iv)

where

$$
\bar{J}=1+\frac{1}{2} \bar{T}_{j j}+\overline{\operatorname{det}\left(\xi_{i, j}\right)}
$$

$$
T_{i j}=\xi_{k, k} \xi_{j, i}-\xi_{k, i} \xi_{j, k}
$$

and also, after a little more manipulation,

By (A 2)

$$
\begin{gathered}
T_{j j}=2\left(\xi_{j} \xi_{k, k}\right)_{, j}-\left(\xi_{j} \xi_{k}\right)_{, j k} . \\
K_{i j} \epsilon_{j p q}=\epsilon_{i l m} \Xi_{l, p} \Xi_{m, q}, \\
K_{i j} \epsilon_{i p q}=\epsilon_{j l m} \Xi_{p, l} \Xi_{q, m} .
\end{gathered}
$$

Equation (A 12) is the relation needed for transforming an integral over a surface $\Sigma$ to an integral over the image $\Sigma^{\xi}$ of $\Sigma$ under the mapping $\mathbf{x} \rightarrow \mathbf{\Xi}$, as in (7.1). Denote the vectorial area elements by $d \mathbf{\Sigma}$ and $d \mathbf{\Sigma}^{\Sigma}$. If $d \mathbf{r}$ and $d \mathbf{s}$ are distinct, infinitesimal vectors tangential to $\Sigma$ at a point $\mathbf{x}$, we may take

and similarly for $d \boldsymbol{\Sigma}^{\xi}$. Thus

$$
d \mathbf{\Sigma}=d \mathbf{r}(\mathbf{x}) \times d \mathbf{s}(\mathbf{x})
$$

$$
\begin{aligned}
\int_{\Sigma \xi} \varphi d \Sigma_{i}^{\xi_{i}} & =\int_{\mathbf{x} \in \Sigma} \varphi(\Xi) \epsilon_{i l m} \Xi_{l, p} d r_{p}(\mathbf{x}) \Xi_{m, q} d s_{q}(\mathbf{x}) \\
& =\int_{\mathbf{x} \in \Sigma} \varphi^{\xi}(\mathbf{x}) K_{i j} \epsilon_{j p q} d r_{p}(\mathbf{x}) d s_{q}(\mathbf{x}) \quad(\text { by A 12) } \\
& =\int_{\Sigma} \varphi^{\xi} K_{i j} d \Sigma_{j} .
\end{aligned}
$$

For an alternative proof, write the left-hand side in the form

$$
\int_{y-\xi}\left(\varphi_{, i}\right)^{\xi} d V^{\xi}
$$

by the divergence theorem, and then use (A 4) and (A 3). Also (A 13), (2.4b) and the fact that $\epsilon_{j m l} \boldsymbol{\Xi}_{p, l m}=0$ show that

$$
K_{i j} \epsilon_{i q p}\left(\varphi_{, i}\right)^{\xi}=\epsilon_{j m l}\left(\varphi^{\xi} \Xi_{p, l}\right)_{, m}
$$

By taking $\varphi=u_{p}+(\boldsymbol{\Omega} \times \mathbf{x})_{p}$ in (A 16), averaging, and recalling (3.1), we get, after a little manipulation,

which is (7.5).

$$
\overline{K_{i j}(\nabla \times \mathbf{u}+2 \Omega)_{i}^{\bar{E}}}=\left[\nabla \times\left(\overline{\mathbf{u}}^{\mathrm{L}}-\mathbf{p}\right)\right]_{j}+2 \Omega_{j},
$$




\section{Appendix B. Derivation of theorem I}

Taking the terms in $(3.2)^{\xi}$ one by one, we have, recalling $(2.14),(2.9)$ and $(2.10 a)$,

$$
\begin{aligned}
\Xi_{j, i}\left(D u_{j} / D t\right)^{\bar{\xi}} & =\Xi_{j, i} \bar{D}^{\mathrm{L}}\left(u_{j}^{\xi}\right)=\bar{D}^{\mathrm{L}}\left(\Xi_{j, i} u_{j}^{\xi}\right)-u_{j}^{\xi}\left\{\left(\bar{D}^{\mathrm{L}} \Xi_{j}\right)_{, i}-\bar{u}_{k, i}^{\mathrm{L}} \Xi_{j, k}\right\} \\
& =\bar{D}^{\mathrm{L}}\left\{\left(\delta_{j i}+\xi_{j, i}\right) u_{j}^{\xi}\right\}-u_{j}^{\xi}\left(u_{j}^{\xi}\right)_{, i}+\bar{u}_{k, i}^{\mathrm{L}}\left(\delta_{j k}+\xi_{j, k}\right) u_{j}^{\xi} .
\end{aligned}
$$

Taking (B 2), defining

$$
\mathrm{P}_{i}^{0}=-\overline{\xi_{j, i} u_{j}^{l}},
$$

noting that this equals $-\overline{\xi_{j, i} u \xi_{j}}$ by (2.7), and recalling (2.1), we have

$$
\overline{\Xi_{j, i} \bar{D}^{\mathrm{L}}\left(u_{j}^{\xi}\right)}=\bar{D}^{\mathrm{I}}\left(\bar{u}_{i}^{\mathrm{L}}-\mathrm{p}_{i}^{0}\right)-\frac{1}{2}\left(\overline{\left.u_{j}^{\xi} u_{j}^{\xi}\right)_{, i}}+\bar{u}_{k, i}^{\mathrm{L}}\left(\bar{u}_{k}^{\mathrm{T}}-\mathrm{p}_{k}^{0}\right)\right. \text {. }
$$

Next, the Coriolis term in $(3.2)^{\frac{t}{5}}$ gives

$$
\begin{aligned}
2 \overline{\Xi_{j, i} \epsilon_{j m n} \Omega_{m} u_{n}^{\xi}}=2 \epsilon_{j m n} \Omega_{m}\left(\overline{\left.\delta_{j i}+\xi_{j, i}\right)\left(\bar{u}_{n}^{\mathrm{I}}+\bar{D}^{\mathrm{L}} \xi_{n}\right)}=2 \epsilon_{i m n} \Omega_{m} \bar{u}_{n}^{\mathrm{L}} \overline{ }\right. & +2 \epsilon_{j m n} \Omega_{m} \overline{\xi_{j, i} \bar{D}^{\mathrm{L}} \xi_{n}},
\end{aligned}
$$

where (2.7) has again been used. The second term

$$
2 \epsilon_{j m n} \Omega_{m} \overline{\xi_{j, i} \bar{D}^{\mathrm{L}} \xi_{n}}=2 \epsilon_{j m n} \Omega_{m}\left\{\overline{\left(\overline{\xi_{j}} \bar{D}^{\mathrm{L}} \xi_{n}\right)_{, i}}-\overline{\xi_{j} \bar{D}^{\mathrm{L} \xi_{n, i}}}-\bar{u}_{v, i}^{\mathrm{L}} \overline{\xi_{j} \xi_{n, p}}\right\} .
$$

Adding half the left-hand side to half the right, exchanging $j$ and $n$ in the middle term within the braces, and changing its sign, we have

$$
\begin{aligned}
2 \epsilon_{j m n} \Omega_{m} \overline{\xi_{j, i} \bar{D}^{\mathrm{L}} \xi_{n}} & =\epsilon_{j m n} \Omega_{m}\left\{\overline{\left(\xi_{j} \bar{D}^{\mathrm{L} \xi_{n}}\right)_{, i}}+\overline{\xi_{n} \bar{D}^{\mathrm{I} \xi_{j, i}}}+\overline{\xi_{j, i} \bar{D}^{\mathrm{I}} \xi_{n}}-\bar{u}_{p, i}^{\mathrm{L}} \overline{\xi_{j} \xi_{n, p}}\right\} \\
& =\epsilon_{j m n} \Omega_{m}\left\{\overline{\left(\xi_{j} u_{n}^{l}\right)_{, i}}+\bar{D}^{\mathrm{L}}\left(\overline{\left.\xi_{n} \xi_{j, i}\right)}-\bar{u}_{p, i}^{\mathrm{L}} \overline{\xi_{j} \xi_{n, p}}\right\}\right.
\end{aligned}
$$

Defining

$$
\mathrm{p}_{i}^{\Omega}=-\epsilon_{j n n} \Omega_{m} \overline{\xi_{j, i} \xi_{n}}=-\overline{\xi_{j, i}(\boldsymbol{\Omega} \times \xi)_{j}},
$$

we see that (B 5) equals

Now (3.1) states that

$$
2\left(\boldsymbol{\Omega} \times \overline{\mathbf{u}}^{\mathrm{L}}\right)_{i}-\left(\overline{\mathbf{u}} \boldsymbol{l} \cdot \boldsymbol{\Omega} \times \xi_{{ }_{i}}-\bar{D}^{\mathrm{L}} \mathrm{p}_{i}^{\Omega}-\bar{u}_{k, i}^{\mathrm{L}} \mathrm{P}_{k}^{\Omega} .\right.
$$

$$
\mathrm{p}_{i}=\mathrm{p}_{i}^{0}+\mathrm{p}_{i}^{\Omega},
$$

so adding (B 4), (B 7), $(\overline{\mathbf{3 . 6}})$ and $\overline{(3.7}$ ) to

gives $(3.8)$.

$$
\overline{\Xi_{j, i} X_{j}^{\xi}}=\overline{\left(\delta_{j i}+\xi_{j, i}\right)\left(\overline{X_{j}^{L}+X_{j}^{T}}\right)}=\bar{X}_{i}^{L}+\overline{\xi_{j, i} X_{j}^{l}}
$$

\section{Appendix C. Proof that $\xi$ returns to zero when the Eulerian disturbance returns to zero, under the circumstances envisaged in $\S 5.3$}

By definition $\bar{S}^{\mathrm{L}}$ and $\bar{Z}^{\mathrm{L}}$ are functions of $x_{2}, x_{3}$ and $t$ but not of $x_{1}$. We fix $t>t_{f}$ and suppress reference to $t$. $\mathrm{By}(5.14)$ the mapping $\left(x_{2}, x_{3}\right) \rightarrow\left(\bar{S}^{\mathrm{L}}, \bar{Z}^{\mathrm{L}}\right)$ is invertible; this together with the relations $D S / D t=D Z / D t=0$ will enable $S$ and $Z$ to be used as measures of particle position in the meridional plane, and hence as a means of relating $\xi_{2}$ and $\xi_{3}$ directly to the Eulerian disturbance fields; mass continuity will then turn out to be sufficient to determine $\xi_{1}$. We shall assume that $\xi(x)$ is a continuous function, and use postulate (vii) of $\S 2$, which asserts invertibility of the mapping

$$
\mathbf{x} \rightarrow \mathbf{x}+\boldsymbol{\xi}(\mathbf{x}) \text {. }
$$


Because $D S / D t=D Z / D t=0, S$ and $Z$ satisfy relations of the type $\bar{S}^{L}=S^{\xi}$ for all $t$, by (2.23), and hence for $t>t_{f}$ in particular (whether or not $\xi$ is then zero). Since also $S=\bar{S}$, by $(5.13)$, and hence $S^{\overline{5}}=(\bar{S})^{\xi}$, we have

similarly

$$
\begin{aligned}
& \bar{S}^{\mathrm{L}}(\mathbf{x})=\bar{S}(\mathbf{x}+\xi) ; \\
& \bar{Z}^{\mathrm{L}}(\mathbf{x})=\bar{Z}(\mathbf{x}+\xi) .
\end{aligned}
$$

For any given $\left(x_{2}, x_{3}\right)=(b, c)$, say, in the meridional plane, define

$$
\langle b, c\rangle=\left\{\mathbf{x} \mid x_{2}=b, x_{3}=c\right\}
$$

the line parallel to the $x_{1}$ axis whose 'meridional' co-ordinates are $(b, c)$. We first prove that $\xi_{2}(\mathbf{x})=\xi_{3}(\mathbf{x})=0$ for all $\mathbf{x} \in\langle b, c\rangle$.

Let the curve $\mathscr{C}$ be the image of the line $\langle b, c\rangle$ under the mapping $\mathbf{x} \rightarrow \mathbf{x}+\boldsymbol{\xi} . \mathscr{C}$ will consist of a single, continuous space curve (as was suggested in figure 1) because of the hypothesis that $\xi$ is a continuous function of $\mathbf{x}$, and thus of $x_{1}$. Now either $\mathscr{C}$ is identical with the line $\langle b, c\rangle$, in which ease $\xi_{2}=\xi_{3}=0$ and there is nothing to prove, or $\mathscr{C}$ is not identical with $\langle b, c\rangle$. In the latter case there must exist a point

$$
\mathbf{x}=\left(a^{\prime}, b^{\prime}, c^{\prime}\right)
$$

on $\mathscr{C}$ whose meridional co-ordinates $\left(b^{\prime}, c^{\prime}\right) \neq(b, c)$ so that the line $\left\langle b^{\prime}, c^{\prime}\right\rangle$ through $\left(b^{\prime}, c^{\prime}\right)$ is distinct from the parallel line $\langle b, c\rangle$. The line $\left\langle b^{\prime}, c^{\prime}\right\rangle$ cannot coincide with $\mathscr{C}$ itself either; for otherwise $\mathscr{C}$ would be parallel to the $x_{1}$ axis, implying $\bar{\xi}_{2}=\xi_{2}=b^{\prime}-b$ and $\bar{\xi}_{3}=c^{\prime}-c$, at least one of which differs from zero, contradicting the basic property $\bar{\xi}=0$ expressed by $(2.7)$. Since therefore $\mathscr{C}$ and $\left\langle b^{\prime}, c^{\prime}\right\rangle$ are not coincident, there exists a point $\left(a^{\prime \prime}, b^{\prime}, c^{\prime}\right)$ which lies on $\left\langle b^{\prime}, c^{\prime}\right\rangle$ but not on $\mathscr{C}$. The inverse image $\mathbf{x}^{0}$ of this point under the mapping $\mathbf{x} \rightarrow \mathbf{x}+\xi$, that is to say the point $\mathbf{x}^{0}$ [unique by postulate (vii)] satisfying

$$
\mathbf{x}^{0}+\xi\left(\mathbf{x}^{0}\right)=\left(a^{\prime \prime}, b^{\prime}, c^{\prime}\right)
$$

therefore does not lie on the inverse image of $\mathscr{C}$, which is $\langle b, c\rangle$ :

Now by $(\mathrm{C} 1 a)$ and $(\mathrm{C} 3)$

$$
\mathbf{x}^{0} \notin\langle b, c\rangle \text {. }
$$

$$
\bar{S}^{\mathrm{L}}\left(\mathbf{x}^{0}\right)=\bar{S}\left(a, b^{\prime \prime}, c^{\prime}\right)=\bar{S}\left(a^{\prime}, b^{\prime}, c^{\prime}\right)
$$

because $\bar{S}(\mathbf{x})$ is independent of $x_{1}$. But the point $\left(a^{\prime}, b^{\prime}, c^{\prime}\right)$ lies on $\mathscr{C}$; therefore its inverse image, $(a, b, c)$ say, lies on $\langle b, c\rangle$, so that by (C $1 a)$ the right-hand side of (C 5) equals $\bar{S}^{\mathrm{L}}(a, b, c)$ for some $a$ (and thus any $a$ ). Therefore

$$
\bar{S}^{\mathrm{L}}\left(\mathbf{x}^{0}\right)=\bar{S}^{\mathrm{L}}(a, b, c),
$$

and similarly for $\bar{Z}^{\mathrm{L}}$ by the same arguments applied to $(\mathrm{C} 1 b)$. Together with (C4) this contradicts the invertibility of the mapping $\left(x_{2}, x_{3}\right) \rightarrow\left(\bar{S}^{\mathrm{L}}, \bar{Z}^{\mathrm{L}}\right)$, and hence shows by reductio ad absurdum that $\mathscr{C}$ cannot after all be distinct from $\langle b, c\rangle$, i.e. that

$$
\xi_{2}(\mathbf{x})=\xi_{3}(\mathbf{x})=0
$$

for all $\mathbf{x} \in\langle b, c\rangle$, and hence for all $\mathbf{x}$ since $b$ and $c$ were chosen arbitrarily in the first place.

Now it may be shown that (C 7) is sufficient to imply

$$
\mathbf{P}=\mathbf{0}
$$


for $t>t_{f}$. However we omit the argument since in fact $\xi_{1}=0$ also, as we shall now show from mass conservation and (2.7). Equation (C 8) will then follow immediately from (3.1).

By (C 7), the Jacobian of the mapping $\mathbf{x} \rightarrow \mathbf{x}+\xi$ reduces exactly to

$$
J=1+\xi_{1,1}
$$

But $\rho(\mathbf{x})=\bar{\rho}(\mathbf{x})$ by (5.13) so, again using (C 7),

$$
\rho^{\xi}=\bar{\rho}\left(x_{1}+\xi_{1}, x_{2}, x_{3}\right)=\bar{\rho}(\mathbf{x}),
$$

since $\bar{\rho}$ is independent of $x_{1}$. Therefore $\rho^{\xi}$ is a mean quantity $\left(\overline{\rho^{\xi}}=\rho^{\xi}\right)$, whence, noting (4.3) and (4.6),

$$
J=\tilde{\rho} / \rho^{\xi}=\overline{\tilde{\rho} / \rho^{\xi}}=\bar{J}=1
$$

by (C 9) and (2.7). This with (C 9) implies that

$$
\xi_{1,1}=0
$$

i.e. that $\xi_{1}$ is independent of $x_{1}$, so that $\xi_{1}=\bar{\xi}_{1}$ and hence, using (2.7) once more,

$$
\xi_{1}=0 \text {. }
$$

\section{REFERENCES}

Andrews, D. G. \& McINTyre, M. E. $1976 a$ Planetary waves in horizontal and vertical shear: the generalized Eliassen-Palm relation and the mean zonal acceleration. J. Atmos. Sci. 33, 2031-2048.

Andrews, D. G. \& McInTyre, M. E. $1976 b$ Planetary waves in horizontal and vertical shear: asymptotic theory for equatorial waves in weak shear. J. Atmos. Sci. 33, 2049 2053.

Avdrews, D. G. \& McIntyre, M. E. $1978 a$ Generalized Eliassen-Palm and Charney-Drazin theorems for waves on axisymmetric flows in compressible atmospheres. J. Atmos. Sci. 35, 175-185.

Andrews, D. G. \& McIntyre, M. E. $1978 b$ On wave-action and its relatives. J. Fluid Mech. $89,647-664$.

Batchelor, G. K. 1967 An Introduction to Fluid Dynamics. Cambridge University Press.

Boyn, J. 1976 The noninteraction of waves with the zonally-averaged flow on a spherical earth and the interrelationships of eddy fluxes of energy, heat and momentum. J. Atmas. Sci. 33, $2285-2291$.

Braginskif, S. I. 1964 Self-excitation of a magnetic field during the motion of a highly conducting fluid. Zh. Eksp. Teor. Fiz. 47, 1084-1098. (English trans. 1965 Sov. Phys. J. Exp. Theor. Phys. 20, 726-735.)

Bretherton, F. P. $1969 a$ Momentum transport by gravity waves. Quart. J. Roy. Met. Soc. 95, 213-243.

Bretherton, F. P. $1969 b$ On the mean motion induced by internal gravity waves. J. Fluid Mech. 36, 785-803.

Bretherton, F. P. 1971 The general linearised theory of wave propagation. Lectures Appl. Math. 13, 61-102. Am. Math. Soc. (See § 6.)

Bretherton, F. P. 1979 Conservation of wave action and angular momentum in a spherical atmosphere. J. Fluid Mech. (to appear).

Bretherton, F. P. \& GarretT, C. J. R. 1968 Wavetrains in inhomogeneous moving media. Proc. Roy. Soc. A 302, 529-554.

Bretherton, F. P. \& Haidvoged, D. B. 1976 Two-dimensional turbulence above topography. J. Fluid Mech. 78, 129-154, equation (53). 
Brillovin, L. 1925 On radiation stresses (in French). Ann. Phys. 4, 528-586.

Brillouin, L. 1936 Radiation pressures and stresses (in French). Rev. Acoust. 5, 99-111. (See also Tensors in Mechanics and Elasticity. Academic, 1964.)

Charkey, J. G. 1973 Planetary fluid dynamics, § IV. In Dymamic Meteorology (ed. P. Morel), pp. 98-351. Reidel.

Charney, J. G. \& Drazin, P. G. 1961 Propagation of planetary-scale disturbances from the lower into the upper atmosphere. J. Geophys. Res. 66, 83-109.

Courant, R. \& Hilbert, D. 1962 Methods of Mathematical Physics, vol. 2. Wiley/Interscience.

Dewar, R. L. 1970 Interaction between hydromagnetic waves and a time-dependent inhomogeneous medium. Phys. Fluids 13, 2710-2720.

ECKarT, C. 1963 Some transformations of the hydrodynamic equations. Phys. Fluids 6, 1037-1041.

Fitassex, A. \& KLeInschmidt. E. 1957 Dynamic meteorology. In Encyclopaedia of Physics, vol. 48, pp. 1-154. Springer.

Eliassen, A. \& PaLM, E. 1961 On the transfer of energy in stationary mountain waves. Geofys. Publ. 22 (3), 1-23.

Fels, S. B. \& LiNDzeN, R. S. 1974 The interaction of thermally excited gravity waves with mean flows. Geophys. Fluid Dyn. 6, 149-191.

FRIEMAN, E. \& RotenberG, M. 1960 On hydromagnetic stability of stationary equilibria. Rev. Mod. Phys. 32, 898-902.

GARRETT, C. J. R. 1968 On the interaction between internal gravity waves and a shear flow. J. Fluid Mech. 34, 711-720.

Geisler, J. E. 1974 A numerical model of the sudden stratospheric warming mechanism. J. Geophys. Res. 79, 4989-4999.

GrImsHAw, R. H. J. 1975 Nonlinear internal gravity waves in a rotating fluid. J. Fluid Mech. 71, 497-512.

Grimshaw, R. H. J. 1977 The modulation of an internal gravity wave paoket, and the resonance with the mean motion. Studies in Appl. Math. 56, 241-266.

Grtmshaw, R. H. J. 1978 Mean flows induced by iriternal gravity wave packets propagating in a shear flow. Submitted to Proc. Roy. Soc. A.

Holton, J. R. 1975 The Dynamic Meteorology of the Stratosphere and Mesosphere. Am. Mot. Soc.

Holton, J. R. \& Dunkerton, T. 1978 On the role of wave transience and dissipation in stratospheric mean flow vacillations. J. Atmos. Sci. 35, 740-744.

Holton, J. R. \& MAss, C. 1976 Stratospheric vacillation cycles. J. Atmos. Sci. 33, 22182225.

Hoskins, B. J. 1974 The role of potential vorticity in symmetric stability and instability. Quart. J. Roy. Met. Soc. 100, 480-482.

JoNes, W. L. 1973 Asymmetric wave-stress tensors and wave spin. J. Fluid Mech. 58, 737747.

Landau, L. D. \& Lifshitz, E. M. 1959 Fluid Mechanics. Pergamon.

LandaU, L. D. \& Lifshitz, E. M. 1975 The Classical Theory of Fields, 4th English edn. Pergamon.

LighthILI, M. J. 1978 a Waves in Fluids. Cambridge University Press.

Liahthrtl, M. J. $1978 b$ Acoustic streaming. Invited Lecture, Inst. Acoust. Spring Conf.

LINDZEN, R. S. \& TSAY, C. Y. 1975 Wave structure of the tropical stratosphere over the Marshall Islands area during 1 April-1 July 1958. J. Atmas, Sci. 32, 2008-2021.

LongueT-Higgins, M. S. \& StewarT, R. W. 1964 Radiation stresses in water waves: a physical discussion, with applications. Deep-Sea Res. 11, 529-562.

MCComas, C. H. \& BRETHERTON, F. P. 1977 Resonant interaction of oceanic internal waves. J. Geophys. Res. 82, 1397-1412.

MoIntyre, M. E. 1973 Mean motions and impulse of a guided intemal gravity wave packet. J. Fluid Mech. 60, 801-811. 
McIntyre, M. E. 1977 Wave transport in stratified, rotating fluids. In Lecture Notes in Phys. vol. 71 (ed. E. A. Spiegel \& J. P. Zahn), pp. 290-314. Springer. (The statement on p. 303, line 27 is wrong; but see equation (5.2) on p. 311.)

MCINTYRE, M. E. 1978 An introduction to the generalized Lagrangian-mean description of wave, mean-flow interaction. Adv. Study Prog. Summer Lecture Notes, N.C.A.R.

Matsuno, T. 1971 A dynamical model of the stratospheric sudden warming. J. Atmos. Sci. $28,1479-1494$.

Moffatt, H. K. 1978 Magnetic Field Generation in Electrically Conducting Fluids. Cambridge University Press.

Moore, D. 1970 The mass transport velocity induced by free oscillations at a single frequency. Geophys. Fluid Dyn. 1, 237-247.

Müller, P. 1976 On the diffusion of momentum and mass by internal gravity waves. J. Fluid Mech. 77, 789-823.

Prierls, R. 1976 The momentum of light in a refracting medium. Proc. Roy. Soc. A 347, $475-491$.

Plumb, R. A. 1975 Momentum transport by the thermal tide in the stratosphere of Venus. Quart. J. Roy. Met. Soc. 101, 763-776.

Plums, R. A. 1977 The interaction of two internal waves with the mean flow: implications for the theory of the quasi-biennial oscillation. J. Atmos. Sci. 34, 1847-1858.

Plumb, R. A. \& McEwan, A. D. 1978 The instability of a forced standing wave in a viscous, stratified fluid: a laboratory analogue of the quasi-biennial oscillation. J. Atmos. Sci. 35, $1827-1839$.

RAYleIGH, LoRD 1896 The Theory of Sound, vol. II, \$ 352. Dover.

RILey, N. 1967 Oscillatory viscous flows, review and extension. J. Inst. Math. Appl. 3, 419434.

Schirchting, H. 1932 Berochnung ebener periodischer Grenzschichtstromungen. Phys. Z. 33, 327.

SowArd, A. M. 1972 A kinematic theory of large magnetic Reynolds number dynamos. Phil. Trans. Roy. Soc. A 272, 431-462.

Soward, A. M. \& RoberTs, P. H. 1976 Recent developments in the theory of the MHD dynamo (in Russian). Magnitnaya Gidrodynamica 1, 3-51.

StuART, J. T. 1966 Double boundary layers in oscillatory viscous flow. J. Fluid Mech. 24, $673-687$.

URYU, M. 1974 Induction and transmission of mean zonal flow by quasi-geostrophic disturbances. J. Met. Soc. Japan 52, 341-363.

Watson, K. M., West, B. J. \& Cohen, B. I. 1976 Coupling of surface and internal gravity waves: a mode coupling model. J. Fluid Mech. 77, 185-208. 